\title{
Higher Homotopy Commutativity of $H$-spaces and the Cyclohedra
}

\author{
Dedicated to Professor Yutaka Hemmi on his sixtieth birthday
}

by

Yusuke KaWAмото

\begin{abstract}
We define higher homotopy commutativity of $H$-spaces using the cyclohedra $\left\{W_{n}\right\}_{n \geq 1}$ constructed by Bott and Taubes. An $H$-space whose multiplication is homotopy commutative of the $n$-th order is called a $B_{n}$-space. We also give combinatorial decompositions of the permuto-associahedra $\left\{K P_{n}\right\}_{n \geq 1}$ introduced by Kapranov into unions of product spaces of cyclohedra. From the decomposition, we have a relation between the $B_{n}$-structures and another notion of higher homotopy commutativity represented by the permuto-associahedra.
\end{abstract}

2010 Mathematics Subject Classification: Primary 55P48, 55P45; Secondary 52B11, $18 \mathrm{D} 10$

Keywords: higher homotopy commutativity, $H$-spaces, cyclohedra, $B_{n}$-spaces, permutoassociahedra.

\section{§1. Introduction}

The concept of higher homotopy commutativity was introduced by Sugawara [26] and Williams [28] in the case of topological monoids. In the definition, Williams used permutohedra, which were introduced by Milgram [20] to construct approximations to iterated loop spaces. The homotopy commutativity of the third order in the sense of Williams is illustrated by the left hexagon in Figure 1.

Later Hemmi-Kawamoto [11] considered another type of higher homotopy commutativity of topological monoids using the resultohedra $\left\{N_{m, n}\right\}_{m, n \geq 1}$ constructed by Gel'fand-Kapranov-Zelevinsky [7]. In particular, we have higher homotopy commutativity represented by the simplices $\left\{\Delta^{m}\right\}_{m \geq 1}$ since $N_{m, 1} \cong \Delta^{m}$

Communicated by T. Ohtsuki. Received October 16, 2012. Revised April 15, 2013.

Y. Kawamoto: Department of Mathematics, National Defense Academy, Yokosuka 239-8686, Japan;

e-mail: yusuke@nda.ac.jp

(C) 2013 Research Institute for Mathematical Sciences, Kyoto University. All rights reserved. 

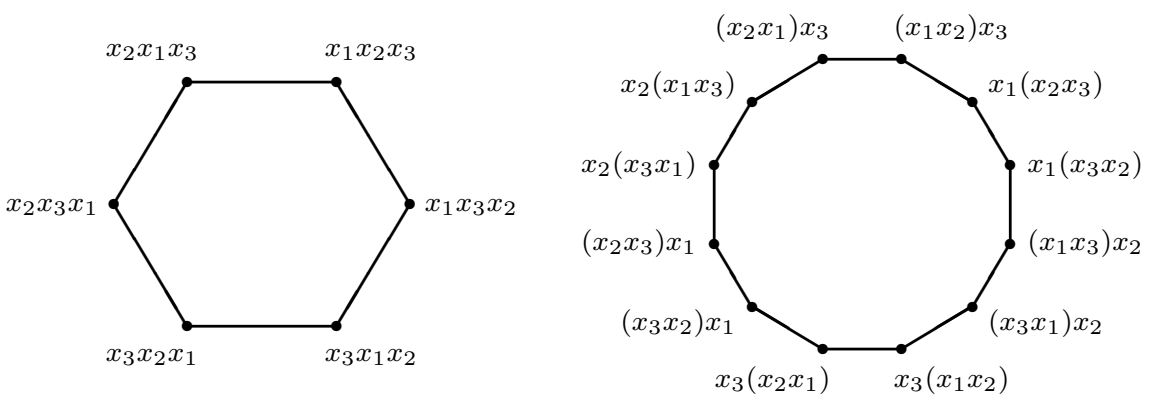

Figure 1. Homotopy commutativity of the third order.

for $m \geq 1$. A $C(n)$-space is a topological monoid with homotopy commutativity of the $n$-th order (see Section 4). From the definition, a topological monoid is a $C(2)$-space if and only if the multiplication is homotopy commutative. The $C(3)-$ structure is illustrated in Figure 11. By Proposition 4.3, $X$ is a $C(\infty)$-space if and only if the classifying space $B X$ is a $T$-space in the sense of Aguadé [1].

In this paper, we show that the $C(n)$-structures can be defined only assuming that multiplication is homotopy associative of the $n$-th order.

According to Sugawara [25], there is a criterion for an $H$-space to have the homotopy type of a topological monoid. His criterion is higher homotopy associativity for multiplication. Later Stasheff [22] expanded the theory of Sugawara, and introduced the concept of $A_{n}$-spaces. An $A_{n}$-space is an $H$-space whose multiplication is homotopy associative of the $n$-th order. When defining $A_{n}$-spaces, he constructed special polytopes $\left\{K_{n}\right\}_{n \geq 1}$ called associahedra.

Bott-Taubes [4] introduced another family $\left\{W_{n}\right\}_{n \geq 1}$ of special polytopes called cyclohedra to study topological descriptions of self-linking invariants of knots. Since the cyclohedra are constructed by combining simplices and associahedra, we can use these polytopes to generalize the $C(n)$-structures to the case of $A_{n}$-spaces.

An $A_{n}$-space with homotopy commutativity of the $n$-th order is called a $B_{n}$-space (see Section 4). From the definition, a $B_{2}$-space is the same as a homotopy commutative $H$-space. Let $X$ be an $A_{3}$-space with a $B_{2}$-structure. Using the associating homotopy $\mu_{3}: K_{3} \times X^{3} \rightarrow X$ and the commuting homotopy $\varphi_{2}: W_{2} \times X^{2} \rightarrow X$, we can define $\widetilde{\varphi}_{3}: \partial W_{3} \times X^{3} \rightarrow X$ illustrated by the left hexagon in Figure 2. Then $X$ is a $B_{3}$-space if and only if $\widetilde{\varphi}_{3}$ extends to $\varphi_{3}: W_{3} \times X^{3} \rightarrow X$. We note that the above hexagon is similar to the one of Mac Lane $[16$, p. $38,(4.5)]$. In this manner, $X$ is called a $B_{n}$-space if there is a family $\left\{\varphi_{i}: W_{i} \times X^{i} \rightarrow X\right\}_{1 \leq i \leq n}$ of maps with the relations stated in Definition 4.4. When $X$ is a topological monoid, $X$ is a $B_{n}$-space if and only if $X$ is a $C(n)$-space. 

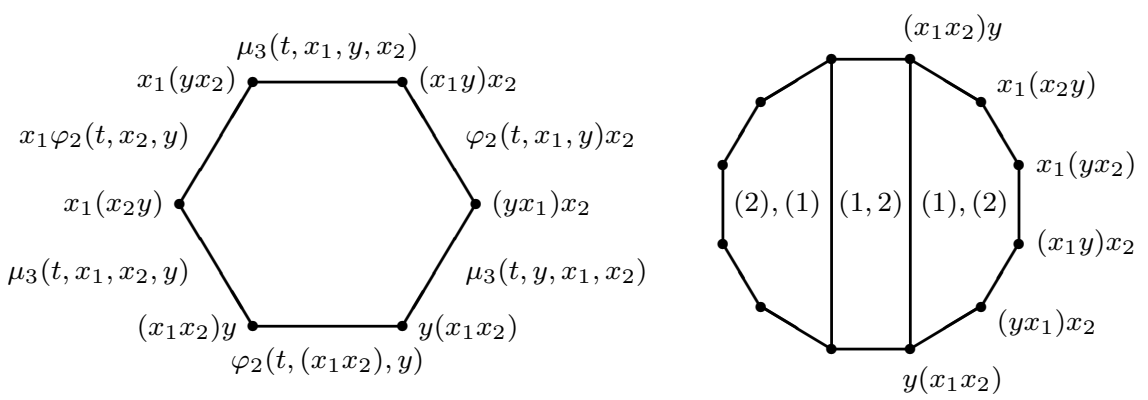

Figure 2. The $B_{3}$-structure on $X$ and the decomposition of $K P_{3}$.

In [9], we also generalized higher homotopy commutativity in the sense of Williams to the case of $A_{n}$-spaces (see the right dodecagon in Figure 1). In the definition, we used the permuto-associahedra $\left\{K P_{n}\right\}_{n \geq 1}$ originally constructed by Kapranov [13] (see Section 3). An $A_{n}$-space with higher homotopy commutativity of this type is called an $A C_{n}$-space.

May [18] introduced the concept of $E_{n}$-space to give a criterion for a space to have the homotopy type of an $n$-fold loop space. The types of higher homotopy commutativity we are considering in this paper are just truncations of $E_{2^{-}}$ structures, just as $A_{n}$-spaces are truncated versions of $E_{1}$-spaces.

According to Hemmi $[8$, p. 108, (5.1)] and Kapranov-Voevodsky [14, Theorem 6.5], permutohedra can be combinatorially decomposed into unions of product spaces of simplices (see [14, p. 245, Figures 14 and 15]). To describe a relation between $B_{n}$-structures and $A C_{n}$-structures, we generalize their result to the case of permuto-associahedra.

We now recall some notation and terminology. Put $\mathbf{n}=(1, \ldots, n) \in \mathbb{N}^{n}$ and

$$
\mathbb{T}^{m}[n]=\left\{\left(t_{1}, \ldots, t_{m}\right) \in \mathbb{N}^{m} \mid t_{1}+\cdots+t_{m}=n\right\} \quad \text { for } m, n \geq 1 .
$$

A subsequence of $\mathbf{n}$ of length $t$ is written as $\alpha=(\alpha(1), \ldots, \alpha(t))$ with $\alpha(1)<$ $\cdots<\alpha(t)$. A partition of $\mathbf{n}$ of type $\left(t_{1}, \ldots, t_{m}\right) \in \mathbb{T}^{m}[n]$ is an ordered sequence $\left(\alpha_{1}, \ldots, \alpha_{m}\right)$ consisting of disjoint subsequences $\alpha_{i}$ of $\mathbf{n}$ of length $t_{i}$ for $1 \leq$ $i \leq m$ with $\alpha_{1} \cup \cdots \cup \alpha_{m}=\mathbf{n}$ as sets. Let $\mathbb{A}_{n}^{\left(t_{1}, \ldots, t_{m}\right)}$ denote the set of all partitions of $\mathbf{n}$ of type $\left(t_{1}, \ldots, t_{m}\right) \in \mathbb{T}^{m}[n]$. For example, $\mathbb{A}_{2}^{(2)}=\{((1,2))\}$, $\mathbb{A}_{3}^{(3)}=\{((1,2,3))\}, \mathbb{A}_{3}^{(1,2)}=\{((1),(2,3)),((2),(1,3)),((3),(1,2))\}$ and $\mathbb{A}_{3}^{(2,1)}=$ $\{((1,2),(3)),((1,3),(2)),((2,3),(1))\}$. Moreover, we see that

$$
\mathbb{A}_{n}^{(1, \ldots, 1)}=\left\{((\sigma(1)), \ldots,(\sigma(n))) \mid \sigma \in \mathscr{S}_{n}\right\} \quad \text { for } n \geq 1,
$$

where $\mathscr{S}_{n}$ denotes the symmetric group on $n$ letters. Put

$$
\mathbb{A}_{n}=\left\{\left(\alpha_{1}, \ldots, \alpha_{m}\right) \in \mathbb{A}_{n}^{\left(t_{1}, \ldots, t_{m}\right)} \mid\left(t_{1}, \ldots, t_{m}\right) \in \mathbb{T}^{m}[n] \text { with } m \geq 1\right\} .
$$


Our result is as follows:

Theorem A. Let $n \geq 2$. There is a family

$$
\left\{\mathscr{D}\left(\alpha_{1}, \ldots, \alpha_{m}\right)\right\}_{\left(\alpha_{1}, \ldots, \alpha_{m}\right) \in \mathbb{A}_{n-1}}
$$

of subspaces of $K P_{n}$ with the following properties:

(1) If $\left(\alpha_{1}, \ldots, \alpha_{m}\right) \in \mathbb{A}_{n-1}^{\left(t_{1}, \ldots, t_{m}\right)}$, then we have an isomorphism

$$
\iota^{\left(\alpha_{1}, \ldots, \alpha_{m}\right)}: W_{m+1} \times K P_{t_{1}} \times \cdots \times K P_{t_{m}} \rightarrow \mathscr{D}\left(\alpha_{1}, \ldots, \alpha_{m}\right) .
$$

(2) $K P_{n}$ decomposes as

$$
K P_{n}=\bigcup_{\left(\alpha_{1}, \ldots, \alpha_{m}\right) \in \mathbb{A}_{n-1}} \mathscr{D}\left(\alpha_{1}, \ldots, \alpha_{m}\right)
$$

In the above theorem, isomorphism of polytopes means affine homeomorphism. The decomposition of $K P_{3}$ is illustrated by the right dodecagon in Figure 2 (see Figure 10 for the decomposition of $\left.K P_{4}\right)$. Then $\mathscr{D}((1,2)) \cong W_{2} \times K P_{2}$ via $\iota^{((1,2))}$ and $\mathscr{D}((\sigma(1)),(\sigma(2))) \cong W_{3} \times K P_{1} \times K P_{1}$ by means of $\iota^{((\sigma(1)),(\sigma(2)))}$ for $\sigma \in \mathscr{S}_{2}$. It is remarkable that the decomposition of $K P_{3}$ also appears in Mac Lane [16, p. 40] and Bar-Natan [2, p. 171, Figure 6].

From Theorem A and an inductive argument, we see that $K P_{n}$ can be decomposed into a union of product spaces of $\left\{W_{i}\right\}_{1 \leq i \leq n}$ in a combinatorial way. Then $W_{n}$ can be regarded as a subspace of $K P_{n}$ via $\iota^{((1), \ldots,(n-1))}: W_{n} \times K P_{1} \times$ $\cdots \times K P_{1} \rightarrow \mathscr{D}((1), \ldots,(n-1)) \subset K P_{n}$

From Theorem A, we have the following result:

Theorem B. If $X$ is a $B_{n}$-space, then $X$ is an $A C_{n}$-space for $n \geq 1$.

The above result generalizes [11, Proposition 4.5] to the case of $A_{n}$-spaces. By Example 4.12, the converse of Theorem B is not true.

This paper is organized as follows: In Section 2, we recall combinatorial properties of the associahedra $\left\{K_{n}\right\}_{n \geq 1}$ and the cyclohedra $\left\{W_{n}\right\}_{n \geq 1}$. In order to prove Theorem A in Section 3, we define a poset $\left(\mathscr{F}_{n}, \preceq_{f}\right)$ describing the faces of $W_{n}$. Then we study the face operators and degeneracy operators of $W_{n}$. In Section 3, we recall the permuto-associahedra $\left\{K P_{n}\right\}_{n \geq 1}$, and give a proof of Theorem A. It is also shown that the degeneracy operators of $K P_{n}$ can be reconstructed from those of $W_{n}$ using Theorem A. Section 4 is devoted to studying higher homotopy commutativity of $A_{n}$-spaces. In the case of topological monoids, we first recall the definition of $C(n)$-spaces (see Definition 4.1 and Remark 4.2). Using cyclohedra instead of simplices, we define $B_{n}$-spaces (see Definition 4.4 and Remark 4.5). It 
is shown that the property of being a $B_{n}$-space is preserved by covering spaces. We also give some examples of $B_{n}$-spaces (see Examples 4.7, 4.8 and 4.12). Then we recall the definition of $A C_{n}$-spaces, and prove Theorem $\mathrm{B}$ using Theorem $\mathrm{A}$.

\section{$\S 2$. Cyclohedra}

We first recall the associahedra $\left\{K_{n}\right\}_{n \geq 1}$ and the cyclohedra $\left\{W_{n}\right\}_{n \geq 1}$ constructed by Stasheff [22] and Bott-Taubes [4], respectively.

Stasheff [22, I, Section 6] constructed the associahedra $\left\{K_{n}\right\}_{n \geq 1}$ in order to define $A_{n}$-spaces (see Section 3). From the construction, the associahedron $K_{n}$ is a polytope of dimension $n-2$ whose faces correspond to meaningful bracketings of the word $x_{1} \cdots x_{n}$ for $n \geq 2$. More precisely, a codimension $t$ face of $K_{n}$ is represented by inserting $t$ pairs of brackets in a meaningful way into the word $x_{1} \cdots x_{n}$ so that any pair of brackets includes at least two elements each of which is $x_{i}$ or a bracketed sequence for $t \geq 1$. In particular, each vertex of $K_{n}$ is represented by one of the meaningful complete ways of bracketing the word $x_{1} \cdots x_{n}$. For convenience, we also put $K_{1}=\{*\}$.

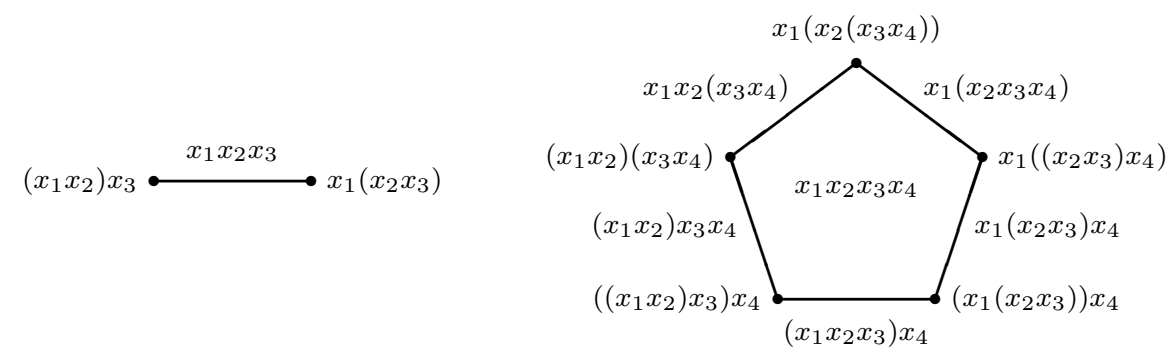

Figure 3. The associahedra $K_{3}$ and $K_{4}$.

Denote the set of all meaningful bracketings of the word $x_{1} \cdots x_{n}$ by $\mathscr{K}_{n}$. Then $\left(\mathscr{K}_{n}, \preceq_{k}\right)$ is a poset (partially ordered set) ordered by defining $\xi \preceq_{k} \xi^{\prime}$ if $\xi^{\prime}$ is obtained from $\xi$ by removing some pairs of brackets or $\xi^{\prime}=\xi$. Let $K_{k}(r, s)$ be the facet (codimension-one face) of $K_{n}$ represented by

$$
x_{1} \cdots x_{k-1}\left(x_{k} \cdots x_{k+s-1}\right) x_{k+s} \cdots x_{n} \in \mathscr{K}_{n} \quad \text { for }(r, s, k) \in \mathbb{K}_{n},
$$

where

$$
\mathbb{K}_{n}=\left\{(r, s, k) \in \mathbb{N}^{3} \mid r, s \geq 2 \text { with } r+s=n+1 \text { and } k \leq r\right\} .
$$

Then the boundary $\partial K_{n}$ is given by

$$
\partial K_{n}=\bigcup_{(r, s, k) \in \mathbb{K}_{n}} K_{k}(r, s) .
$$


According to Stasheff [22, I, Section 2], $K_{k}(r, s) \cong K_{r} \times K_{s}$ via a face operator $\partial_{k}(r, s): K_{r} \times K_{s} \rightarrow K_{k}(r, s)$ for $(r, s, k) \in \mathbb{K}_{n}$ and there is a family of degeneracy operators $\left\{\theta_{j}: K_{n} \rightarrow K_{n-1}\right\}_{1 \leq j \leq n}$.

Later Bott-Taubes [4, Section 1] introduced another family $\left\{W_{n}\right\}_{n \geq 1}$ of special complexes closely related to the associahedra. According to Stasheff [24, p. 58], $W_{n}$ is called a cyclohedron for $n \geq 1$.

Stasheff [24, Section 10] and Markl [17, Section 1] reconstructed $W_{n}$ as the convex hull of a finite set of points in $\mathbb{R}^{n}$, and gave a poset representing all the faces of $W_{n}$. By their results, $W_{n}$ is a polytope of dimension $n-1$ whose faces correspond to meaningful bracketings of the string $x_{1} \cdots x_{n}$ arranged on a circle for $n \geq 1$ (see also Devadoss [6, Section 1]). Such bracketings are called cyclic bracketings. In particular, $W_{n}$ is represented by the string $x_{1} \cdots x_{n}$ without brackets, and a codimension $t$ face of $W_{n}$ is represented by a cyclic bracketing of the string $x_{1} \cdots x_{n}$ including just $t$ pairs of brackets for $t \geq 1$. In this manner, the vertices of $W_{n}$ correspond to all complete ways of cyclic bracketings of the string $x_{1} \cdots x_{n}$.
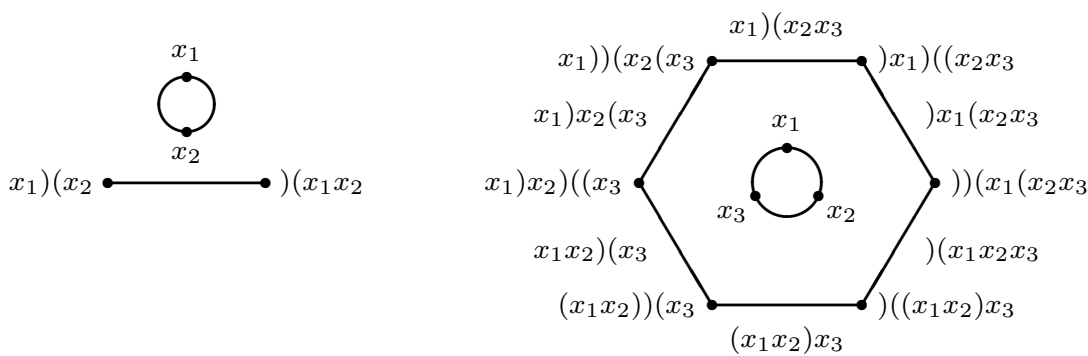

Figure 4 . The cyclic bracketings of the strings $x_{1} x_{2}$ and $x_{1} x_{2} x_{3}$.

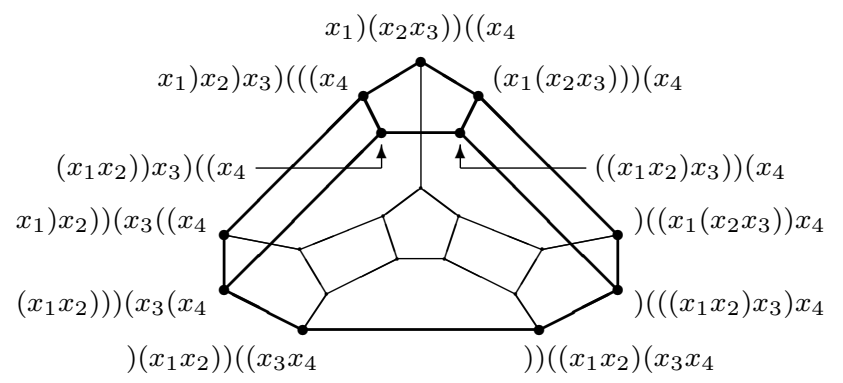

Figure 5. The cyclic bracketings of the string $x_{1} x_{2} x_{3} x_{4}$.

We denote the set of all cyclic bracketings of the string $x_{1} \cdots x_{n}$ by $\mathscr{W}_{n}$. Then $\left(\mathscr{W}_{n}, \preceq_{w}\right)$ is a poset, where the poset structure $\preceq_{w}$ is defined in a similar way to 
the one of $\left(\mathscr{K}_{n}, \preceq_{k}\right)$. Put

$$
\begin{aligned}
& \mathbb{W}_{n}=\left\{(r, s, k) \in \mathbb{N}^{3} \mid r, s \geq 2 \text { with } r+s=n+1 \text { and } k \leq r-1\right\}, \\
& \mathbb{W}_{n}^{\prime}=\left\{(r, s, k) \in \mathbb{N}^{3} \mid r \geq 2 \text { with } r+s=n+1 \text { and } k \leq r\right\} .
\end{aligned}
$$

Let $W_{k}(r, s)$ and $W_{k}^{\prime}(r, s)$ denote the facets of $W_{n}$ represented by

$$
\begin{aligned}
x_{1} \cdots x_{k-1}\left(x_{k} \cdots x_{k+s-1}\right) x_{k+s} \cdots x_{n} \in \mathscr{W}_{n} & \text { for }(r, s, k) \in \mathbb{W}_{n}, \\
\left.x_{1} \cdots x_{k-1}\right) x_{k} \cdots x_{k+s-2}\left(x_{k+s-1} \cdots x_{n} \in \mathscr{W}_{n}\right. & \text { for }(r, s, k) \in \mathbb{W}_{n}^{\prime},
\end{aligned}
$$

respectively. Then the boundary $\partial W_{n}$ is given by

$$
\partial W_{n}=\bigcup_{(r, s, k) \in \mathbb{W}_{n}} W_{k}(r, s) \cup \bigcup_{(r, s, k) \in \mathbb{W}_{n}^{\prime}} W_{k}^{\prime}(r, s) .
$$

Remark 2.1. We have a simple proof of the well-known result that $\left|\operatorname{vert}\left(K_{n}\right)\right|=$ $\frac{1}{n}\left(\begin{array}{c}2 n-2 \\ n-1\end{array}\right)$ using $W_{n}$, where $\operatorname{vert}(Q)$ denotes the set of all vertices of a polytope $Q$ and $|S|$ is the number of elements of a set $S$. Let $v$ be a vertex of $W_{n}$. Then $v$ is represented by one of the complete ways of cyclic bracketing of the string $x_{1} \cdots x_{n}$. Replacing $x_{i}$ with $\bullet$ for $1 \leq i \leq n-1$ and removing $x_{n}$ and all the closing brackets ")" from $v$, we have a bijection between vert $\left(W_{n}\right)$ and the set of all permutations of $\{\overbrace{(, \ldots, t,}^{n-1}, \overbrace{\bullet, \ldots, \bullet}^{n-1}\}$. For example, the vertices of $W_{4}$ represented by $\left.\left.\left(x_{1} x_{2}\right)\right)\right)\left(x_{3}\left(x_{4}\right.\right.$ and $\left.\left.\left.x_{1}\right) x_{2}\right)\right)\left(x_{3}\left(\left(x_{4}\right.\right.\right.$ correspond to $(\bullet \bullet(\bullet)$ and $\bullet \bullet(\bullet(($, respectively. Then $\left|\operatorname{vert}\left(W_{n}\right)\right|=\left(\begin{array}{c}2 n-2 \\ n-1\end{array}\right)$, which implies the required result since $\left|\operatorname{vert}\left(W_{n}\right)\right|=$ $n\left|\operatorname{vert}\left(K_{n}\right)\right|$.

We next give an alternative description of the poset $\left(\mathscr{W}_{n}, \preceq_{w}\right)$ to be used in the proof of Theorem A in Section 3.

Consider the rectangle $\mathbb{E}_{n}=[0, n-1] \times I$ for $n \geq 2$. A lattice path in $\mathbb{E}_{n}$ is a map $\ell:[0, n] \rightarrow \mathbb{E}_{n}$ such that $\ell(0)=(0,0), \ell(n)=(n-1,1)$ and if we write $\ell(s)=\left(\ell_{1}(s), \ell_{2}(s)\right)$ for $s \in[0, n]$, then $\ell(i+t)$ is either $\left(\ell_{1}(i)+t, \ell_{2}(i)\right)$ or $\left(\ell_{1}(i), \ell_{2}(i)+t\right)$ for $0 \leq i<n$ and $t \in I$. We denote the set of all lattice paths in $\mathbb{E}_{n}$ by $\mathscr{L}_{n}$.

In $\mathbb{E}_{n}$, we label the interval $[i-1, i] \times\{j\}$ by $x_{i}$ for $1 \leq i \leq n-1$ and $j=0,1$, and the interval $\{i\} \times I$ by $y$ for $0 \leq i \leq n-1$ as in Figure 6 . Then each lattice

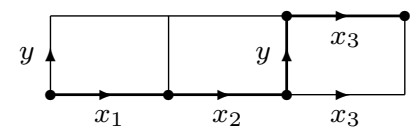

Figure 6. The lattice path $\ell=x_{1} x_{2} y x_{3}$. 
path $\ell \in \mathscr{L}_{n}$ is labeled by a word $x_{1} \cdots x_{i-1} y x_{i} \cdots x_{n-1}$ for some $i$ with $1 \leq i \leq n$. In this label of $\ell$, the symbol $x_{i}$ means the horizontal unit move from the line $x=i-1$ to the line $x=i$ for $1 \leq i \leq n-1$, and $y$ is the vertical move between the lines $y=0$ and $y=1$. For example, the lattice path $\ell \in \mathscr{L}_{4}$ in Figure 6 is labeled by $x_{1} x_{2} y x_{3}$.

Put

$$
\mathbb{H}^{m}[n]=\left\{\left(h_{1}, \ldots, h_{m}\right) \in\left(\mathbb{Z}^{+}\right)^{m} \mid h_{1}+\cdots+h_{m}=n\right\} \quad \text { for } m, n \geq 1,
$$

where $\mathbb{Z}^{+}=\{h \in \mathbb{Z} \mid h \geq 0\}$.

Let $\xi \in \mathscr{W}_{n}$ be such that $x_{n}$ is covered by just $t$ pairs of brackets for $t \geq 0$. Then we can write

$$
\left.\left.\left.\left.\xi=\xi_{1}\right) \xi_{2}\right) \cdots\right) \xi_{t}\right) \xi_{t+1}\left(\xi _ { t + 2 } \left(\cdots \left(\xi _ { 2 t } \left(\xi_{2 t+1} x_{n}\right.\right.\right.\right.
$$

where $\xi_{j}$ is a meaningful bracketing of the word $x_{h_{1}+\cdots+h_{j-1}+1} \cdots x_{h_{1}+\cdots+h_{j}}$ for $1 \leq j \leq 2 t+1$ and $\left(h_{1}, \ldots, h_{2 t+1}\right) \in \mathbb{H}^{2 t+1}[n-1]$ with $h_{j}+h_{2 t+2-j}>0$ for $1 \leq j \leq t$

We now define $\mathscr{F}_{n}=\left\{f(\xi) \mid \xi \in \mathscr{W}_{n}\right\}$, where

$$
f(\xi)=\left(\xi_{1}\left(\xi_{2}\left(\cdots\left(\xi_{t}\left[\xi_{t+1} \mid y\right] \xi_{t+2}\right) \cdots\right) \xi_{2 t}\right) \xi_{2 t+1}\right)
$$

if $\xi \in \mathscr{W}_{n}$ is written as in $(2.3)$. Then $\left(\mathscr{F}_{n}, \preceq_{f}\right)$ is a poset ordered by defining $f(\xi) \preceq_{f} f\left(\xi^{\prime}\right)$ if $\xi \preceq_{w} \xi^{\prime}$ for $\xi, \xi^{\prime} \in \mathscr{W}_{n}$.

Remark 2.2. Let $\xi \in \mathscr{W}_{n}$ be written as in (2.3). From the definition, we have the following relations:

(1) $f(\xi) \prec_{f}\left(\xi_{1}\left(\cdots\left(\xi_{i} \xi_{i+1}\left(\cdots\left(\xi_{t}\left[\xi_{t+1} \mid y\right] \xi_{t+2}\right) \cdots\right) \xi_{2 t+1-i} \xi_{2 t+2-i}\right) \cdots\right) \xi_{2 t+1}\right)$ for $1 \leq i \leq t-1$.

(2) $f(\xi) \prec_{f}\left(\xi_{1}\left(\cdots\left(\xi_{t-1}\left[\xi_{t} \xi_{t+1} \xi_{t+2} \mid y\right] \xi_{t+3}\right) \cdots\right) \xi_{2 t+1}\right)$.

(3) If $\xi_{i}^{\prime}$ is obtained from $\xi_{i}$ by removing some pair of brackets or $\xi_{i}^{\prime}=\xi_{i}$ for $1 \leq i \leq 2 t+1$, then $f(\xi) \preceq f\left(\xi_{1}^{\prime}\left(\cdots\left(\xi_{t}^{\prime}\left[\xi_{t+1}^{\prime} \mid y\right] \xi_{t+2}^{\prime}\right) \cdots\right) \xi_{2 t+1}^{\prime}\right)$.

Since $f:\left(\mathscr{W}_{n}, \preceq_{w}\right) \rightarrow\left(\mathscr{F}_{n}, \preceq_{f}\right)$ is an isomorphism of posets, we can assume that the faces of $W_{n}$ are labeled by $\left(\mathscr{F}_{n}, \preceq_{f}\right)$. Recall that $W_{n}$ is represented by $x_{1} \cdots x_{n} \in \mathscr{W}_{n}$. Then it is labeled by $f\left(x_{1} \cdots x_{n}\right)=\left[x_{1} \cdots x_{n-1} \mid y\right] \in \mathscr{F}_{n}$. By $(2.1)$ and (2.2), the facets $W_{k}(r, s)$ and $W_{k}^{\prime}(r, s)$ are labeled by

$$
\left[x_{1} \cdots x_{k-1}\left(x_{k} \cdots x_{k+s-1}\right) x_{k+s} \cdots x_{n-1} \mid y\right] \in \mathscr{F}_{n} \quad \text { for }(r, s, k) \in \mathbb{W}_{n}
$$

and

$$
\left(x_{1} \cdots x_{k-1}\left[x_{k} \cdots x_{k+s-2} \mid y\right] x_{k+s-1} \cdots x_{n-1}\right) \in \mathscr{F}_{n} \quad \text { for }(r, s, k) \in \mathbb{W}_{n}^{\prime} \text {, }
$$


respectively. In this manner, a vertex of $W_{n}$ is labeled by a meaningful complete way of bracketing of some lattice path $\ell \in \mathscr{L}_{n}$.

The cyclohedra $W_{n}$ whose faces are labeled by $\left(\mathscr{F}_{n}, \preceq_{f}\right)$ for $n=2,3$ and 4 are illustrated in Figures 7 and 8. For simplicity, we denote $[\emptyset \mid y]$ by $y$, and omit
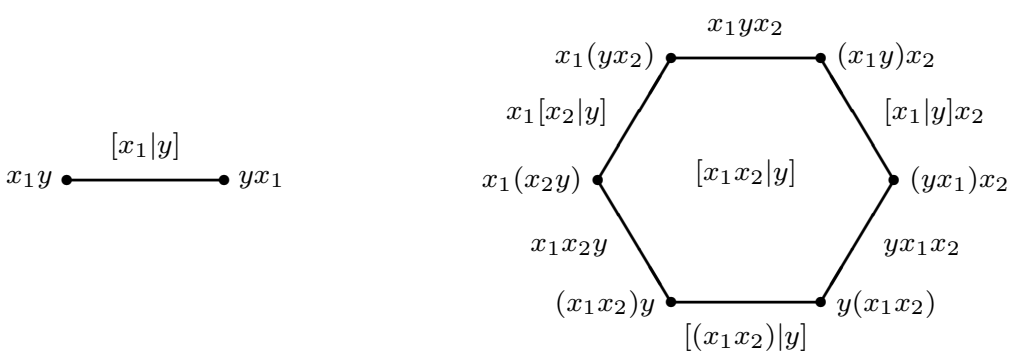

Figure 7. The cyclohedra $W_{2}$ and $W_{3}$.

the outermost pair of brackets. Then $W_{2}$ labeled by $\left[x_{1} \mid y\right]$ is the left interval in Figure 7, which represents a commuting homotopy between $x_{1} y$ and $y x_{1}$.

When $n=3$, the cyclohedron $W_{3}$ labeled by $\left[x_{1} x_{2} \mid y\right]$ is illustrated by the right hexagon in Figure 7 . The bottom edge labeled by $\left[\left(x_{1} x_{2}\right) \mid y\right]$ represents a commuting homotopy between $\left(x_{1} x_{2}\right) y$ and $y\left(x_{1} x_{2}\right)$, and the next left edge labeled by $x_{1} x_{2} y$ is an associating homotopy between $\left(x_{1} x_{2}\right) y$ and $x_{1}\left(x_{2} y\right)$. The next edge labeled by $x_{1}\left[x_{2} \mid y\right]$ is regarded as a commuting homotopy between $x_{1}\left(x_{2} y\right)$ and $x_{1}\left(y x_{2}\right)$.

Remark 2.3. The cyclohedra $\left\{W_{n}\right\}_{n \geq 1}$ realizing the posets $\left\{\left(\mathscr{F}_{n}, \preceq_{f}\right)\right\}_{n \geq 1}$ are closely related to the commuto-associahedra $\left\{C A_{n}\right\}_{n \geq 1}$ introduced by Bar-Natan [2, Sections 5 and 6] (see also [3, Section 4] and [6, p. 73, 4.2]). In particular, the 2-skeleton of $W_{n}$ is a subspace of $C A_{n}$ for $n \geq 1$.

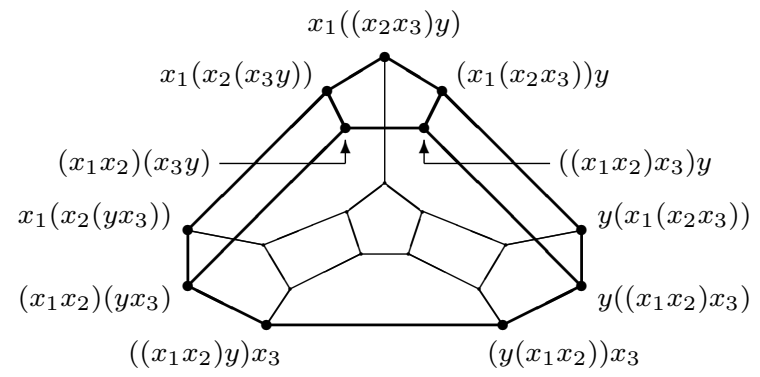

Figure 8. The cyclohedron $W_{4}$. 
Since the set of all faces of $W_{k}(r, s)$ is described by the poset $\left(\mathscr{F}_{r} \times \mathscr{K}_{s}, \sqsubseteq\right)$, it follows that $W_{k}(r, s) \cong W_{r} \times K_{s}$ for $(r, s, k) \in \mathbb{W}_{n}$, where the poset structure of $\mathscr{F}_{r} \times \mathscr{K}_{s}$ is given by defining $(\lambda, \xi) \sqsubseteq\left(\lambda^{\prime}, \xi^{\prime}\right)$ if $\lambda \preceq_{f} \lambda^{\prime}$ and $\xi \preceq_{k} \xi^{\prime}$. In a similar way, we see that $W_{k}^{\prime}(r, s) \cong K_{r} \times W_{s}$ for $(r, s, k) \in \mathbb{W}_{n}^{\prime}$. Define face operators $\varepsilon_{k}(r, s): W_{r} \times K_{s} \rightarrow W_{k}(r, s)$ and $\varepsilon_{k}^{\prime}(r, s): K_{r} \times W_{s} \rightarrow W_{k}^{\prime}(r, s)$ of $W_{n}$ by using these isomorphisms. From the construction, we have the following proposition:

Proposition 2.4. The face operators $\left\{\varepsilon_{k}(r, s)\right\}_{(r, s, k) \in \mathbb{W}_{n}},\left\{\varepsilon_{k}^{\prime}(r, s)\right\}_{(r, s, k) \in \mathbb{W}_{n}^{\prime}}$ and $\left\{\partial_{k}(r, s)\right\}_{(r, s, k) \in \mathbb{K}_{n}}$ satisfy the following relations:

$$
\begin{aligned}
& \varepsilon_{k}(r, s)\left(\varepsilon_{l}(p, q)(a, b), c\right) \\
& = \begin{cases}\varepsilon_{l+s-1}(p+s-1, q)\left(\varepsilon_{k}(p, s)(a, c), b\right) & \text { if } k \leq l-1, \\
\varepsilon_{l}(p, q+s-1)\left(a, \partial_{k-l+1}(q, s)(b, c)\right) & \text { if } l \leq k \leq l+q-1, \\
\varepsilon_{l}(p+s-1, q)\left(\varepsilon_{k-q+1}(p, s)(a, c), b\right) & \text { if } k \geq l+q,\end{cases} \\
& \text { for }(r, s, k) \in \mathbb{W}_{n} \text { and }(p, q, l) \in \mathbb{W}_{r} \text {; } \\
& \varepsilon_{k}(r, s)\left(\varepsilon_{l}^{\prime}(p, q)(a, b), c\right) \\
& = \begin{cases}\varepsilon_{l+s-1}^{\prime}(p+s-1, q)\left(\partial_{k}(p, s)(a, c), b\right) & \text { if } k \leq l-1, \\
\varepsilon_{l}^{\prime}(p, q+s-1)\left(a, \varepsilon_{k-l+1}(q, s)(b, c)\right) & \text { if } l \leq k \leq l+q-2, \\
\varepsilon_{l}^{\prime}(p+s-1, q)\left(\partial_{k-q+2}(p, s)(a, c), b\right) & \text { if } k \geq l+q-1,\end{cases} \\
& \text { for }(r, s, k) \in \mathbb{W}_{n} \text { and }(p, q, l) \in \mathbb{W}_{r}^{\prime} \text {; } \\
& \varepsilon_{k}^{\prime}(r, s)\left(a, \varepsilon_{l}^{\prime}(p, q)(b, c)\right)=\varepsilon_{k+l-1}^{\prime}(r+p-1, q)\left(\partial_{k}(r, p)(a, b), c\right) \\
& \text { for }(r, s, k) \in \mathbb{W}_{n}^{\prime} \text { and }(p, q, l) \in \mathbb{W}_{s}^{\prime} \text {. }
\end{aligned}
$$

We now explain the proposition in the case of $n=3$ and 4 .

In the right hexagon of Figure 7 , the bottom edge labeled by $\left[\left(x_{1} x_{2}\right) \mid y\right]$ is isomorphic to $W_{2} \times K_{2}$ by means of the face operator $\varepsilon_{1}(2,2)$, and the edge labeled by $x_{1} x_{2} y$ is isomorphic to $K_{3} \times W_{1}$ via $\varepsilon_{3}^{\prime}(3,1)$. The intersection of these two edges is a vertex which is the image of $\left(\varepsilon_{2}^{\prime}(2,1)(*, *), *\right)$ under $\varepsilon_{1}(2,2)$ and of $\left(\partial_{1}(2,2)(*, *), *\right)$ under $\varepsilon_{3}^{\prime}(3,1)$.

The next left vertex is the intersection of the two edges labeled by $x_{1} x_{2} y$ and $x_{1}\left[x_{2} \mid y\right]$, the image of $\left(\partial_{2}(2,2)(*, *), *\right)$ in $K_{3} \times W_{1}$ under $\varepsilon_{3}^{\prime}(3,1)$ and of $\left(*, \varepsilon_{2}^{\prime}(2,1)(*, *)\right)$ in $K_{2} \times W_{2}$ under $\varepsilon_{2}^{\prime}(2,2)$. The next vertex, the intersection of the two edges $x_{1}\left[x_{2} \mid y\right]$ and $x_{1} y x_{2}$, is the image of $\left(*, \varepsilon_{1}^{\prime}(2,1)(*, *)\right)$ in $K_{2} \times W_{2}$ under $\varepsilon_{2}^{\prime}(2,2)$ and of $\left(\partial_{2}(2,2)(*, *), *\right)$ in $K_{3} \times W_{1}$ under $\varepsilon_{2}^{\prime}(3,1)$.

In the case of $W_{4}$, the front hexagon, the right rectangle and the top pentagon of Figure 8 are labeled by $\left[\left(x_{1} x_{2}\right) x_{3} \mid y\right],\left[\left(x_{1} x_{2} x_{3}\right) \mid y\right]$ and $x_{1} x_{2} x_{3} y$, respectively. Then the facet $\left[\left(x_{1} x_{2}\right) x_{3} \mid y\right]$ is isomorphic to $W_{3} \times K_{2}$ via $\varepsilon_{1}(3,2)$, while the facet 
labeled by $\left[\left(x_{1} x_{2} x_{3}\right) \mid y\right]$ is isomorphic to $W_{2} \times K_{3}$ via $\varepsilon_{1}(2,3)$. The intersection of these two facets is an edge which is the image $\left(\varepsilon_{1}(2,2)(a, *), *\right)$ under $\varepsilon_{1}(3,2)$ and of $\left(a, \partial_{1}(2,2)(*, *)\right)$ under $\varepsilon_{1}(2,3)$ for $a \in W_{2}$.

The facet $x_{1} x_{2} x_{3} y$ is isomorphic to $K_{4} \times W_{1}$ via $\varepsilon_{4}^{\prime}(4,1)$, and the intersection of $\left[\left(x_{1} x_{2}\right) x_{3} \mid y\right]$ and $x_{1} x_{2} x_{3} y$ is an edge which is the image $\left(\varepsilon_{3}^{\prime}(3,1)(b, *), *\right)$ under $\varepsilon_{1}(3,2)$ and of $\left(\partial_{1}(3,2)(b, *), *\right)$ under $\varepsilon_{4}^{\prime}(4,1)$ for $b \in K_{3}$.

In a similar way to the proof of [20, Lemma 4.5], we have the following proposition:

Proposition 2.5. There are degeneracy operators $\left\{\delta_{j}: W_{n} \rightarrow W_{n-1}\right\}_{1 \leq j \leq n-1}$ and $\delta_{n}: W_{n} \rightarrow K_{n-1}$ with the following relations:

$$
\begin{aligned}
& \delta_{n} \varepsilon_{1}(2, n-1)(a, b)=b \\
& \delta_{k} \varepsilon_{k}(n-1,2)(a, *)=\delta_{k+1} \varepsilon_{k}(n-1,2)(a, *)=a \quad \text { for } 1 \leq k \leq n-2 \\
& \delta_{j} \varepsilon_{k}(r, s)(a, b)= \begin{cases}\varepsilon_{k-1}(r-1, s)\left(\delta_{j}(a), b\right) & \text { if } 1 \leq j \leq k-1 \\
\varepsilon_{k}(r, s-1)\left(a, \theta_{j-k+1}(b)\right) & \text { if } k \leq j \leq k+s-1, \\
\varepsilon_{k}(r-1, s)\left(\delta_{j-s+1}(a), b\right) & \text { if } k+s \leq j \leq n-1, \\
\partial_{k}(r-1, s)\left(\delta_{r}(a), b\right) & \text { if } j=n,\end{cases}
\end{aligned}
$$$$
\text { for }(r, s, k) \in \mathbb{W}_{n} \text { excluding (2.7) and (2.8); }
$$

(2.10) $\delta_{n-1} \varepsilon_{1}^{\prime}(2, n-1)(*, b)=\delta_{1} \varepsilon_{2}^{\prime}(2, n-1)(*, b)=b$;

(2.11) $\quad \delta_{n} \varepsilon_{k}^{\prime}(n, 1)(a, *)=\theta_{k}(a) \quad$ for $1 \leq k \leq n$;

(2.12) $\quad \delta_{n} \varepsilon_{k}^{\prime}(n-1,2)(a, b)=a \quad$ for $1 \leq k \leq n-1$;

$$
\delta_{j} \varepsilon_{k}^{\prime}(r, s)(a, b)= \begin{cases}\varepsilon_{k-1}^{\prime}(r-1, s)\left(\theta_{j}(a), b\right) & \text { if } 1 \leq j \leq k-1, \\ \varepsilon_{k}^{\prime}(r, s-1)\left(a, \delta_{j-k+1}(b)\right) & \text { if } k \leq j \leq k+s-2, \\ \varepsilon_{k}^{\prime}(r-1, s)\left(\theta_{j-s+2}(a), b\right) & \text { if } k+s-1 \leq j \leq n-1, \\ \partial_{k}(r, s-1)\left(a, \delta_{s}(b)\right) & \text { if } j=n,\end{cases}
$$

Proof. We prove the case of $\left\{\delta_{j}\right\}_{1 \leq j \leq n-1}$ by induction on $n$. When $n=2$, we put $\delta_{1}(a)=*$. Let $n>2$, and assume inductively that $\left\{\delta_{j}: W_{n^{\prime}} \rightarrow W_{n^{\prime}-1}\right\}_{1 \leq j \leq n^{\prime}-1}$ are constructed for any $n^{\prime}<n$.

We now define $\left\{\widetilde{\delta}_{j}: \partial W_{n} \rightarrow W_{n-1}\right\}_{1 \leq j \leq n-1}$ by (2.8)-(2.10) and (2.13). Since $W_{n}$ is regarded as the cone of $\partial W_{n}$, if $a \in W_{n}$, then we can write $a=(b, t)$ with $b \in \partial W_{n}$ and $t \in I$. Set $\widetilde{\delta}_{j}(b)=(c, u)$ with $c \in \partial W_{n-1}$ and $u \in I$. Let $\delta_{j}: W_{n} \rightarrow W_{n-1}$ be defined by $\delta_{j}(a)=(c, t u)$. Then $\left\{\delta_{j}\right\}_{1 \leq j \leq n-1}$ satisfies the required conditions. In the case of $\delta_{n}: W_{n} \rightarrow K_{n-1}$, the proof is similar. 


\section{§3. Permuto-associahedra}

We recall the permuto-associahedra $\left\{K P_{n}\right\}_{n \geq 1}$ constructed by Kapranov [13] and Reiner-Ziegler [21].

Kapranov [13, Section 2] constructed a family $\left\{K P_{n}\right\}_{n \geq 1}$ of special complexes such that $K P_{n}$ is homeomorphic to the ball of dimension $n-1$ for $n \geq 1$. Later Reiner-Ziegler [21, Theorem 2] reconstructed $K P_{n}$ as the convex hull of a finite set of points in $\mathbb{R}^{n}$ (see also Ziegler [29, Definition 9.13 and Example 9.14]). The polytopes $\left\{K P_{n}\right\}_{n \geq 1}$ are called permuto-associahedra.

From the construction, there is a natural way of describing all the faces of $K P_{n}$. Let $\mathbb{K P}_{n}=\left\{\left(\alpha_{1}, \ldots, \alpha_{m}\right) \in \mathbb{A}_{n} \mid m \geq 2\right\}$. By the above results, a facet of $K P_{n}$ is represented by $\left(\alpha_{1}, \ldots, \alpha_{m}\right) \in \mathbb{K}_{n}$, and a codimension-two face is represented by inserting a pair of brackets in $\left(\alpha_{1}, \ldots, \alpha_{m}\right) \in \mathbb{K} \mathbb{P}_{n}$ as

$$
\left(\alpha_{1}, \ldots, \alpha_{k-1},\left(\alpha_{k}, \ldots, \alpha_{k+s-1}\right), \alpha_{k+s}, \ldots, \alpha_{m}\right) \quad \text { for }(m-s+1, s, k) \in \mathbb{K}_{m} .
$$

In general, a codimension $t$ face of $K P_{n}$ is represented by inserting $t-1$ pairs of brackets in a meaningful way into some $\left(\alpha_{1}, \ldots, \alpha_{m}\right) \in \mathbb{K}_{n}$ for $t \geq 1$. In this manner, each vertex of $K P_{n}$ corresponds to a meaningful complete way of bracketing of some $\left(\alpha_{1}, \ldots, \alpha_{n}\right) \in \mathbb{A}_{n}^{(1, \ldots, 1)}$.

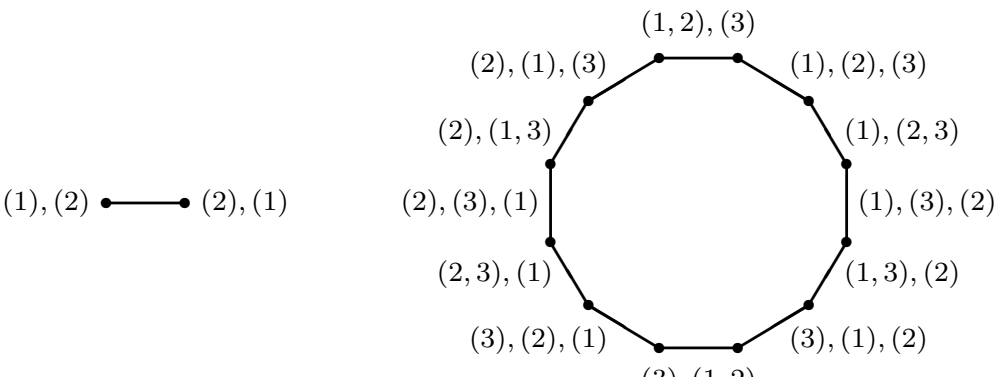

$(3),(1,2)$

Figure 9. The permuto-associahedra $K P_{2}$ and $K P_{3}$.

Let $K P\left(\alpha_{1}, \ldots, \alpha_{m}\right)$ denote the facet represented by $\left(\alpha_{1}, \ldots, \alpha_{m}\right) \in \mathbb{K} \mathbb{P}_{n}$. Then the boundary $\partial K P_{n}$ is given by

$$
\partial K P_{n}=\bigcup_{\left(\alpha_{1}, \ldots, \alpha_{m}\right) \in \mathbb{K} \mathbb{P}_{n}} K P\left(\alpha_{1}, \ldots, \alpha_{m}\right) .
$$

By [13, p. 139] and [9, Proposition 2.1], $K P\left(\alpha_{1}, \ldots, \alpha_{m}\right) \cong K_{m} \times K P_{t_{1}} \times \cdots \times K P_{t_{m}}$ via a face operator $\varepsilon^{\left(\alpha_{1}, \ldots, \alpha_{m}\right)}: K_{m} \times K P_{t_{1}} \times \cdots \times K P_{t_{m}} \rightarrow K P\left(\alpha_{1}, \ldots, \alpha_{m}\right)$ for $\left(\alpha_{1}, \ldots, \alpha_{m}\right) \in \mathbb{A}_{n}^{\left(t_{1}, \ldots, t_{m}\right)}$.

To prove Theorem A, we show the following lemma (cf. [22, I, Proposition 25]): 
Lemma 3.1. There is a family $\left\{\eta_{m}: W_{3} \times K_{m} \rightarrow W_{m+1}\right\}_{m \geq 2}$ of homeomorphisms with the following relations:

$$
\begin{aligned}
& \eta_{m}\left(\varepsilon_{1}(2,2)(a, *), b\right)=\varepsilon_{1}(2, m)(a, b), \\
& \eta_{m}\left(a, \partial_{k}(r, s)(b, c)\right)=\varepsilon_{k}(r+1, s)\left(\eta_{r}(a, b), c\right) \quad \text { for }(r, s, k) \in \mathbb{K}_{m} .
\end{aligned}
$$

Proof. We work by induction on $m$. When $m=2$, we define $\eta_{2}(a, *)=a$ for $a \in W_{3}$. Let $m>2$, and assume inductively that $\left\{\eta_{j}\right\}_{2 \leq j<m}$ are constructed.

We now define $\widetilde{\eta}_{m}: \mathscr{V}_{m} \rightarrow W_{m+1}$ by (3.2) and (3.3), where $\mathscr{V}_{m}=W_{1}(2,2) \times$ $K_{m} \cup W_{3} \times \partial K_{m} \subset W_{3} \times K_{m}$. Then $\mathscr{V}_{m}$ is homeomorphic to the ball of dimension $m-1$, and the image of $\widetilde{\eta}_{m}$ is given by

$$
\widetilde{\eta}_{m}\left(\mathscr{V}_{m}\right)=\bigcup_{(r, s, k) \in \mathbb{W}_{m+1}} W_{k}(r, s) .
$$

Let $\eta_{m}: W_{3} \times K_{m} \rightarrow W_{m+1}$ be defined by $\eta_{m}(b, t)=\left(\widetilde{\eta}_{m}(b), t\right)$ with $b \in \mathscr{V}_{m}$ and $t \in I$ since $W_{3} \times K_{m}$ and $W_{m+1}$ are homeomorphic to $\mathscr{V}_{m} \times I$ and $\widetilde{\eta}_{m}\left(\mathscr{V}_{m}\right) \times I$, respectively. Then $\left\{\eta_{j}\right\}_{2 \leq j \leq m}$ satisfy the required relations.

Proof of Theorem A. We work by induction on $n$. When $n=2$, put $\mathscr{D}((1))=W_{2}$ and define $\iota^{((1))}: W_{2} \times K P_{1} \rightarrow \mathscr{D}((1))$ by $\iota^{((1))}(a, *)=a$. Since $K P_{2}=W_{2}=I$, the result is clear.

Let $n>2$, and assume inductively that the result is proved for any $n^{\prime}<n$.

We first define a complex $\mathscr{U}_{n}$ with the properties of Theorem A. Put $\mathscr{U}_{n}=$ $W_{1}(2,2) \times K P_{n-1} \cup W_{3} \times \partial K P_{n-1}$. Then $\mathscr{U}_{n}$ is homeomorphic to the ball of dimension $n-1$. Let $\iota^{((1, \ldots, n-1))}: W_{2} \times K P_{n-1} \rightarrow \mathscr{D}((1, \ldots, n-1))$ be defined by $\iota^{((1, \ldots, n-1))}(a, b)=\left(\varepsilon_{1}(2,2)(a, *), b\right)$, where $\mathscr{D}((1, \ldots, n-1))=W_{1}(2,2) \times K P_{n-1}$ $\subset \mathscr{U}_{n}$. If $\left(\alpha_{1}, \ldots, \alpha_{m}\right) \in \mathbb{A}_{n-1}^{\left(t_{1}, \ldots, t_{m}\right)}$ with $m \geq 2$, then $\iota^{\left(\alpha_{1}, \ldots, \alpha_{m}\right)}: W_{m+1} \times K P_{t_{1}}$ $\times \cdots \times K P_{t_{m}} \rightarrow \mathscr{D}\left(\alpha_{1}, \ldots, \alpha_{m}\right)$ is defined by

$$
\iota^{\left(\alpha_{1}, \ldots, \alpha_{m}\right)}\left(\eta_{m}(a, b), c_{1}, \ldots, c_{m}\right)=\left(a, \varepsilon^{\left(\alpha_{1}, \ldots, \alpha_{m}\right)}\left(b, c_{1}, \ldots, c_{m}\right)\right)
$$

where $\mathscr{D}\left(\alpha_{1}, \ldots, \alpha_{m}\right)=W_{3} \times K P\left(\alpha_{1}, \ldots, \alpha_{m}\right) \subset \mathscr{U}_{n}$ and $\eta_{m}: W_{3} \times K_{m} \rightarrow W_{m+1}$ denotes the homeomorphism of Lemma 3.1. By (3.1), we have

$$
\mathscr{U}_{n}=\bigcup_{\left(\alpha_{1}, \ldots, \alpha_{m}\right) \in \mathbb{A}_{n-1}} \mathscr{D}\left(\alpha_{1}, \ldots, \alpha_{m}\right) .
$$

To see $\mathscr{U}_{n}=K P_{n}$, we show that there is a family

$$
\left\{\mathscr{U}\left(\alpha_{1}, \ldots, \alpha_{m}\right)\right\}_{\left(\alpha_{1}, \ldots, \alpha_{m}\right) \in \mathbb{K} \mathbb{P}_{n}}
$$

of subspaces of $\partial \mathscr{U}_{n}$ with the following properties: 
(1) If $\left(\alpha_{1}, \ldots, \alpha_{m}\right) \in \mathbb{A}_{n}^{\left(t_{1}, \ldots, t_{m}\right)}$ with $m \geq 2$, then we have an isomorphism

$$
\varepsilon^{\left(\alpha_{1}, \ldots, \alpha_{m}\right)}: K_{m} \times K P_{t_{1}} \times \cdots \times K P_{t_{m}} \rightarrow \mathscr{U}\left(\alpha_{1}, \ldots, \alpha_{m}\right) .
$$

(2) $\partial \mathscr{U}_{n}$ decomposes as

$$
\partial \mathscr{U}_{u}=\bigcup_{\left(\alpha_{1}, \ldots, \alpha_{m}\right) \in \mathbb{K}_{n}} \mathscr{U}\left(\alpha_{1}, \ldots, \alpha_{m}\right) .
$$

Put

$$
\begin{array}{r}
\widetilde{\mathscr{D}}\left(\alpha_{1}, \ldots, \alpha_{m}\right)=\iota^{\left(\alpha_{1}, \ldots, \alpha_{m}\right)}\left(\bigcup_{(r, s, k) \in \mathbb{W}_{m+1}^{\prime}} W_{k}^{\prime}(r, s) \times K P_{t_{1}} \times \cdots \times K P_{t_{m}}\right) \subset \partial \mathscr{U}_{n} \\
\\
\quad \text { for }\left(\alpha_{1}, \ldots, \alpha_{m}\right) \in \mathbb{A}_{n-1}^{\left(t_{1}, \ldots, t_{m}\right)} .
\end{array}
$$

Since

$$
\eta_{m}\left(\bigcup_{(r, s, k) \in \mathbb{W}_{3}^{\prime}} W_{k}^{\prime}(r, s) \times K_{m}\right)=\bigcup_{(r, s, k) \in \mathbb{W}_{m+1}^{\prime}} W_{k}^{\prime}(r, s)
$$

by Lemma 3.1, we have

$$
\partial \mathscr{U}_{n}=\bigcup_{\left(\alpha_{1}, \ldots, \alpha_{m}\right) \in \mathbb{A}_{n-1}} \widetilde{\mathscr{D}}\left(\alpha_{1}, \ldots, \alpha_{m}\right) .
$$

Given $\left(\alpha_{1}, \ldots, \alpha_{m}\right) \in \mathbb{A}_{n}^{\left(t_{1}, \ldots, t_{m}\right)}$ with $m \geq 2$, we have $\alpha_{k}\left(t_{k}\right)=n$ for some $k$ with $1 \leq k \leq m$. When $t_{k}=1$, define $\varepsilon^{\left(\alpha_{1}, \ldots, \alpha_{m}\right)}: K_{m} \times K P_{t_{1}} \times \cdots \times K P_{t_{k-1}} \times$ $\{*\} \times K P_{t_{k+1}} \times \cdots \times K P_{t_{m}} \rightarrow \partial \mathscr{U}_{n}$ by

$$
\begin{aligned}
\varepsilon^{\left(\alpha_{1}, \ldots, \alpha_{m}\right)}\left(a, c_{1}, \ldots, c_{k-1}, *, c_{k+1}, \ldots, c_{m}\right) \\
=\iota^{\left(\gamma_{1}, \ldots, \gamma_{m-1}\right)}\left(\varepsilon_{k}^{\prime}(m, 1)(a, *), c_{1}, \ldots, c_{k-1}, c_{k+1}, \ldots, c_{m}\right),
\end{aligned}
$$

where $\left(\gamma_{1}, \ldots, \gamma_{m-1}\right) \in \mathbb{A}_{n-1}^{\left(t_{1}, \ldots, t_{k-1}, t_{k+1}, \ldots, t_{m}\right)}$ is given by

$$
\gamma_{i}(s)= \begin{cases}\alpha_{i}(s) & \text { if } 1 \leq i \leq k-1 \\ \alpha_{i+1}(s) & \text { if } k \leq i \leq m-1\end{cases}
$$

If $t_{k} \geq 2$, then

$$
K P_{t_{k}}=\bigcup_{\left(\beta_{1}, \ldots, \beta_{r}\right) \in \mathbb{A}_{t_{k}-1}} \mathscr{D}\left(\beta_{1}, \ldots, \beta_{r}\right)
$$

by inductive hypothesis, where

$$
\begin{aligned}
\mathscr{D}\left(\beta_{1}, \ldots, \beta_{r}\right)=\iota^{\left(\beta_{1}, \ldots, \beta_{r}\right)}\left(W_{r+1} \times K P_{u_{1}} \times \cdots \times K P_{u_{r}}\right) \subset K P_{t_{k}} \\
\\
\quad \text { for }\left(\beta_{1}, \ldots, \beta_{r}\right) \in \mathbb{A}_{t_{k}-1}^{\left(u_{1}, \ldots, u_{r}\right)} .
\end{aligned}
$$


Let $\varepsilon^{\left(\alpha_{1}, \ldots, \alpha_{m}\right)}: K_{m} \times K P_{t_{1}} \times \cdots \times K P_{t_{m}} \rightarrow \partial \mathscr{U}_{n}$ be defined by

$$
\begin{aligned}
& \varepsilon^{\left(\alpha_{1}, \ldots, \alpha_{m}\right)}\left(a, c_{1}, \ldots, c_{k-1}, \iota \iota^{\left(\beta_{1}, \ldots, \beta_{r}\right)}\left(b, d_{1}, \ldots, d_{r}\right), c_{k+1}, \ldots, c_{m}\right) \\
& =\iota^{\left(\gamma_{1}, \ldots, \gamma_{m+r-1}\right)}\left(\varepsilon_{k}^{\prime}(m, r+1)(a, b), c_{1}, \ldots, c_{k-1}, d_{1}, \ldots, d_{r}, c_{k+1}, \ldots, c_{m}\right),
\end{aligned}
$$

where $\left(\gamma_{1}, \ldots, \gamma_{m+r-1}\right) \in \mathbb{A}_{n-1}^{\left(t_{1}, \ldots, t_{k-1}, u_{1}, \ldots, u_{r}, t_{k+1}, \ldots, t_{m}\right)}$ is given by

$$
\gamma_{i}(s)= \begin{cases}\alpha_{i}(s) & \text { if } 1 \leq i \leq k-1 \\ \alpha_{k} \beta_{i-k+1}(s) & \text { if } k \leq i \leq k+r-1 \\ \alpha_{i-r+1}(s) & \text { if } k+r \leq i \leq m+r-1\end{cases}
$$

Put

$$
\begin{array}{r}
\mathscr{U}\left(\alpha_{1}, \ldots, \alpha_{m}\right)=\varepsilon^{\left(\alpha_{1}, \ldots, \alpha_{m}\right)}\left(K_{m} \times K P_{t_{1}} \times \cdots \times K P_{t_{m}}\right) \subset \partial \mathscr{U}_{n} \\
\\
\quad \text { for }\left(\alpha_{1}, \ldots, \alpha_{m}\right) \in \mathbb{A}_{n}^{\left(t_{1}, \ldots, t_{m}\right)} .
\end{array}
$$

Then

$$
\partial \mathscr{U}_{n}=\bigcup_{\left(\alpha_{1}, \ldots, \alpha_{m}\right) \in \mathbb{K}_{n}} \mathscr{U}\left(\alpha_{1}, \ldots, \alpha_{m}\right)
$$

by (3.5). This completes the proof of Theorem A.

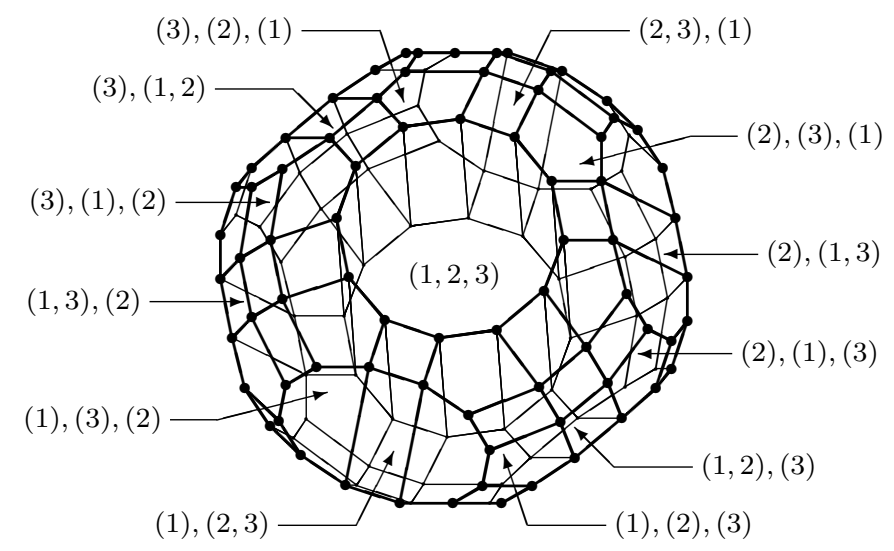

Figure 10. The decomposition of $K P_{4}$.

Remark 3.2. Assume that $\left(\alpha_{1}, \ldots, \alpha_{m}\right) \in \mathbb{A}_{n-1}^{\left(t_{1}, \ldots, t_{m}\right)}$ with $m \geq 2$ and $\left(\beta_{1}, \ldots, \beta_{r}\right)$ $\in \mathbb{A}_{t_{k}}^{\left(u_{1}, \ldots, u_{r}\right)}$ with $r \geq 2$. Then by (3.4) and [9, Proposition 2.1], we have the 
following relations:

$$
\begin{aligned}
& \iota^{\left(\alpha_{1}, \ldots, \alpha_{m}\right)}\left(a, c_{1}, \ldots, c_{k-1}, \varepsilon^{\left(\beta_{1}, \ldots, \beta_{r}\right)}\left(b, d_{1}, \ldots, d_{r}\right), c_{k+1}, \ldots, c_{m}\right) \\
& =\iota^{\left(\gamma_{1}, \ldots, \gamma_{m+r-1}\right)}\left(\varepsilon_{k}(m+1, r)(a, b), c_{1}, \ldots, c_{k-1}, d_{1}, \ldots, d_{r}, c_{k+1}, \ldots, c_{m}\right),
\end{aligned}
$$

where $\left(\gamma_{1}, \ldots, \gamma_{m+r-1}\right) \in \mathbb{A}_{n-1}^{\left(t_{1}, \ldots, t_{k-1}, u_{1}, \ldots, u_{r}, t_{k+1}, \ldots, t_{m}\right)}$ is defined by (3.6).

According to Hemmi-Kawamoto [9, Proposition 2.3], there is a family $\left\{\omega_{j}: K P_{n} \rightarrow K P_{n-1}\right\}_{1 \leq j \leq n}$ of degeneracy operators of $K P_{n}$. From Theorem A and an inductive argument, we can reconstruct $\left\{\omega_{j}\right\}_{1 \leq j \leq n}$ using the degeneracy operators $\left\{\delta_{j}\right\}_{1 \leq j \leq n}$ of $W_{n}$.

When $n=2$, we put $\omega_{j}(a)=*$ for $j=1,2$. Assume inductively that $\left\{\omega_{j}: K P_{n^{\prime}} \rightarrow K P_{n^{\prime}-1}\right\}_{1 \leq j \leq n^{\prime}}$ are constructed for any $n^{\prime}<n$. Let $\left(\alpha_{1}, \ldots, \alpha_{m}\right) \in$ $\mathbb{A}_{n-1}^{\left(t_{1}, \ldots, t_{m}\right)}$.

We first consider the case of $1 \leq j \leq n-1$. Then $\alpha_{k}(t)=j$ for some $k, t$ with $1 \leq k \leq m$ and $1 \leq t \leq t_{k}$. If $t_{k} \geq 2$, then $\omega_{j}: K P_{n} \rightarrow K P_{n-1}$ is defined by

$$
\omega_{j} \iota^{\left(\alpha_{1}, \ldots, \alpha_{m}\right)}\left(a, c_{1}, \ldots, c_{m}\right)=\iota^{\left(\widetilde{\alpha}_{1}, \ldots, \widetilde{\alpha}_{m}\right)}\left(a, c_{1}, \ldots, c_{k-1}, \omega_{t}\left(c_{k}\right), c_{k+1}, \ldots, c_{m}\right),
$$

where $\left(\widetilde{\alpha}_{1}, \ldots, \widetilde{\alpha}_{m}\right) \in \mathbb{A}_{n-1}^{\left(t_{1}, \ldots, t_{k-1}, t_{k}-1, t_{k+1}, \ldots, t_{m}\right)}$ is given by

$$
\widetilde{\alpha}_{k}(s)= \begin{cases}\alpha_{k}(s) & \text { if } \alpha_{k}(s)<j \\ \alpha_{k}(s+1)-1 & \text { if } \alpha_{k}(s) \geq j\end{cases}
$$

and

$$
\widetilde{\alpha}_{i}(s)=\left\{\begin{array}{ll}
\alpha_{i}(s) & \text { if } \alpha_{i}(s)<j, \\
\alpha_{i}(s)-1 & \text { if } \alpha_{i}(s)>j,
\end{array} \quad \text { for } 1 \leq i \leq m \text { with } i \neq k .\right.
$$

When $t_{k}=1$, we put

$$
\begin{aligned}
\omega_{j} \iota^{\left(\alpha_{1}, \ldots, \alpha_{m}\right)}(a, & \left.c_{1}, \ldots, c_{m}\right) \\
& =\iota^{\left(\widetilde{\alpha}_{1}, \ldots, \widetilde{\alpha}_{k-1}, \widetilde{\alpha}_{k+1}, \ldots, \widetilde{\alpha}_{m}\right)}\left(\delta_{k}(a), c_{1}, \ldots, c_{k-1}, c_{k+1}, \ldots, c_{m}\right),
\end{aligned}
$$

where $\left(\widetilde{\alpha}_{1}, \ldots, \widetilde{\alpha}_{k-1}, \widetilde{\alpha}_{k+1}, \ldots, \widetilde{\alpha}_{m}\right) \in \mathbb{A}_{n-1}^{\left(t_{1}, \ldots, t_{k-1}, t_{k+1}, \ldots, t_{m}\right)}$ is given by (3.8).

In the case of $\omega_{n}: K P_{n} \rightarrow K P_{n-1}$, we define $\omega_{n} \iota^{((1, \ldots, n-1))}(a, c)=c$ and $\omega_{n} \iota^{\left(\alpha_{1}, \ldots, \alpha_{m}\right)}\left(a, c_{1}, \ldots, c_{m}\right)=\varepsilon^{\left(\alpha_{1}, \ldots, \alpha_{m}\right)}\left(\delta_{m+1}(a), c_{1}, \ldots, c_{m}\right) \quad$ for $m \geq 2$.

\section{$\S 4$. Higher homotopy commutativity}

Let $\Delta^{m}$ denote the $m$-simplex

$$
\Delta^{m}=\left\{\left(t_{0}, \ldots, t_{m}\right) \in\left(\mathbb{R}^{+}\right)^{m+1} \mid t_{0}+\cdots+t_{m}=1\right\} \quad \text { for } m \geq 0,
$$

where $\mathbb{R}^{+}=\{t \in \mathbb{R} \mid t \geq 0\}$. Then we have face operators $\left\{\partial_{k}: \Delta^{m-1} \rightarrow\right.$ $\left.\Delta^{m}\right\}_{0 \leq k \leq m}$ and degeneracy operators $\left\{\sigma_{j}: \Delta^{m} \rightarrow \Delta^{m-1}\right\}_{1 \leq j \leq m}$ (cf. [8, p. 109]). 
Definition 4.1. Let $n \geq 1$. A topological monoid $X$ is called a $C(n)$-space if there is a family $\left\{\psi_{i}: \Delta^{i-1} \times X^{i} \rightarrow X\right\}_{1 \leq i \leq n}$ of maps with the following relations:

$$
\begin{aligned}
& \psi_{1}(*, y)=y \\
& \psi_{i}\left(\partial_{k}(a), x_{1}, \ldots, x_{i-1}, y\right) \\
& = \begin{cases}x_{1} \psi_{i-1}\left(a, x_{2}, \ldots, x_{i-1}, y\right) & \text { if } k=0, \\
\psi_{i-1}\left(a, x_{1}, \ldots,\left(x_{k} x_{k+1}\right), \ldots, x_{i-1}, y\right) & \text { if } 0<k<i-1, \\
\psi_{i-1}\left(a, x_{1}, \ldots, x_{i-2}, y\right) x_{i-1} & \text { if } k=i-1 ;\end{cases} \\
& \psi_{i}\left(a, x_{1}, \ldots, x_{j-1}, *, x_{j+1}, \ldots, x_{i-1}, y\right) \\
& =\psi_{i-1}\left(\sigma_{j}(a), x_{1}, \ldots, x_{j-1}, x_{j+1}, \ldots, x_{i-1}, y\right) \quad \text { for } 1 \leq j \leq i-1 ; \\
& \psi_{i}\left(a, x_{1}, \ldots, x_{i-1}, *\right)=x_{1} \cdots x_{i-1} \text {. }
\end{aligned}
$$

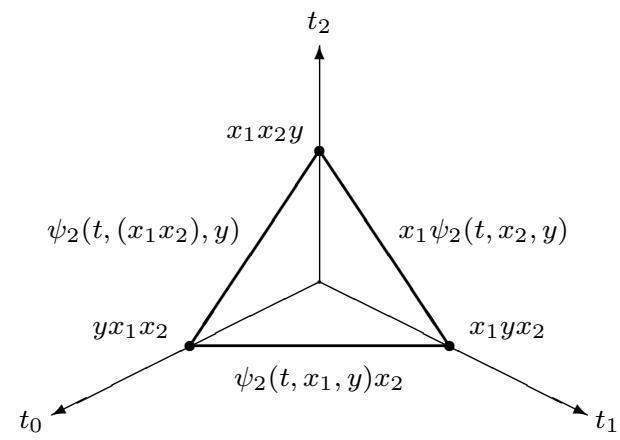

Figure 11. The $C(3)$-structure on $X$.

Remark 4.2. By Definition 4.1, a $C(n)$-space is the same as a $C_{1}(n)$-space in the sense of Hemmi-Kawamoto [11, Definition 4.3] for $n \geq 1$.

A $C(1)$-space is just a topological monoid. Since $\psi_{2}\left(\partial_{0}(1), x, y\right)=x y$ and $\psi_{2}\left(\partial_{1}(1), x, y\right)=y x$ for $x, y \in X$, a topological monoid $X$ is a $C(2)$-space if and only if the multiplication of $X$ is homotopy commutative. Any abelian topological monoid is a $C(\infty)$-space whose $C(\infty)$-structure $\left\{\psi_{i}\right\}_{i \geq 1}$ is given by

$$
\psi_{i}\left(a, x_{1}, \ldots, x_{i-1}, y\right)=x_{1} \cdots x_{i-1} y \quad \text { for } i \geq 1 .
$$

In particular, Eilenberg-Mac Lane spaces are $C(\infty)$-spaces (cf. [23, Corollary 13.10]).

According to Aguadé [1, p. 939], a space $Y$ is called a $T$-space if

$$
\Omega Y \rightarrow \operatorname{Map}\left(S^{1}, Y\right) \stackrel{e}{\rightarrow} Y
$$

is fiber homotopy equivalent to the trivial fibration, where $\Omega Y$ is the based loop 
space of $Y$ and $e: \operatorname{Map}\left(S^{1}, Y\right) \rightarrow Y$ denotes evaluation at the base point. While an $H$-space is always a $T$-space, the converse is not true.

Let $\widetilde{\Omega} Y$ denote the based loop space of $Y$ in the sense of Moore defined by

$$
\widetilde{\Omega} Y=\left\{\alpha:[0, r] \rightarrow Y \mid r \in \mathbb{R}^{+} \text {and } \alpha(0)=\alpha(r)=*\right\}
$$

(cf. [23, Definition 4.1]).

By Remark 4.2, we have the following proposition:

Proposition 4.3 ([11, Corollary 1.1]). A connected topological monoid $X$ is a $C(\infty)$-space if and only if the classifying space $B X$ is a T-space. In particular, if $Y$ is an $H$-space, then $\widetilde{\Omega} Y$ is a $C(\infty)$-space.

Stasheff [22, I, Section 2] defined $A_{n}$-spaces using the associahedra $\left\{K_{i}\right\}_{1 \leq i \leq n}$. An $A_{n}$-form on a space $X$ is a family of maps $\left\{\mu_{i}: K_{i} \times X^{i} \rightarrow X\right\}_{1 \leq i \leq n}$ with the following relations:

$$
\begin{aligned}
& \mu_{1}(*, x)=x \\
& \mu_{i}\left(\partial_{k}(r, s)(a, b), x_{1}, \ldots, x_{i}\right) \\
& =\mu_{r}\left(a, x_{1}, \ldots, x_{k-1}, \mu_{s}\left(b, x_{k}, \ldots, x_{k+s-1}\right), x_{k+s}, \ldots, x_{i}\right) \quad \text { for }(r, s, k) \in \mathbb{K}_{i} ; \\
& \begin{aligned}
\mu_{i}\left(a, x_{1}, \ldots, x_{j-1}, *, x_{j+1}, \ldots, x_{i}\right) \\
\quad=\mu_{i-1}\left(\theta_{j}(a), x_{1}, \ldots, x_{j-1}, x_{j+1}, \ldots, x_{i}\right) \quad \text { for } 1 \leq j \leq i .
\end{aligned}
\end{aligned}
$$

A space with an $A_{n}$-form is called an $A_{n}$-space for $n \geq 1$. From the definition, an $A_{1}$-space is just a space. Since $\mu_{2}(*, x, *)=\mu_{2}(*, *, x)=x$ for $x \in X$, $\mu_{3}\left(\partial_{1}(2,2)(*, *), x_{1}, x_{2}, x_{3}\right)=\left(x_{1} x_{2}\right) x_{3}$ and $\mu_{3}\left(\partial_{2}(2,2)(*, *), x_{1}, x_{2}, x_{3}\right)=x_{1}\left(x_{2} x_{3}\right)$ for $x_{1}, x_{2}, x_{3} \in X$, we see that an $A_{2}$-space and an $A_{3}$-space are the same as an $H$-space and a homotopy associative $H$-space, respectively.

If there is a family $\left\{\mu_{i}\right\}_{i \geq 1}$ of maps such that $\left\{\mu_{i}\right\}_{1 \leq i \leq n}$ is an $A_{n}$-form on $X$ for any $n \geq 1$, then $X$ is called an $A_{\infty}$-space. By [23, Theorem 11.4], $X$ is an $A_{\infty}$-space if and only if $X \simeq \widetilde{\Omega}(B X)$.

Using the cyclohedra $\left\{W_{i}\right\}_{1 \leq i \leq n}$, we generalize Definition 4.1 to the case of $A_{n}$-spaces.

Definition 4.4. Let $n \geq 1$. Assume that $X$ is an $A_{n}$-space with an $A_{n}$-form $\left\{\mu_{i}\right\}_{1 \leq i \leq n}$. Then $X$ is called a $B_{n}$-space if there is a family of maps $\left\{\varphi_{i}: W_{i} \times X^{i} \rightarrow\right.$ $X\}_{1 \leq i \leq n}$ with the following relations:

$$
\begin{aligned}
& \varphi_{1}(*, y)=y \\
& \varphi_{i}\left(\varepsilon_{k}(r, s)(a, b), x_{1}, \ldots, x_{i-1}, y\right) \\
& \quad=\varphi_{r}\left(a, x_{1}, \ldots, x_{k-1}, \mu_{s}\left(b, x_{k}, \ldots, x_{k+s-1}\right), x_{k+s}, \ldots, x_{i-1}, y\right)
\end{aligned}
$$$$
\text { for }(r, s, k) \in \mathbb{W}_{i} \text {; }
$$ 


$$
\begin{array}{ll}
\varphi_{i}\left(\varepsilon_{k}^{\prime}(r, s)(a, b), x_{1}, \ldots, x_{i-1}, y\right) & \\
=\mu_{r}\left(a, x_{1}, \ldots, x_{k-1}, \varphi_{s}\left(b, x_{k}, \ldots, x_{k+s-2}, y\right), x_{k+s-1}, \ldots, x_{i-1}\right) & \text { for }(r, s, k) \in \mathbb{W}_{i}^{\prime} ; \\
& \\
\varphi_{i}\left(a, x_{1}, \ldots, x_{j-1}, *, x_{j+1}, \ldots, x_{i-1}, y\right) & \\
\quad=\varphi_{i-1}\left(\delta_{j}(a), x_{1}, \ldots, x_{j-1}, x_{j+1}, \ldots, x_{i-1}, y\right) & \text { for } 1 \leq j \leq i-1 ; \\
\varphi_{i}\left(a, x_{1}, \ldots, x_{i-1}, *\right)=\mu_{i-1}\left(\delta_{n}(a), x_{1}, \ldots, x_{i-1}\right) . &
\end{array}
$$

Remark 4.5. (1) A $B_{1}$-space is just a space. Since $\varphi_{2}\left(\varepsilon_{2}^{\prime}(2,1)(*, *), x, y\right)=x y$ and $\varphi_{2}\left(\varepsilon_{1}^{\prime}(2,1)(*, *), x, y\right)=y x$ for $x, y \in X$, a $B_{2}$-space is the same as a homotopy commutative $H$-space.

(2) When $X$ is a topological monoid, $X$ is a $B_{n}$-space if and only if $X$ is a $C(n)$-space.

Let $X$ and $Y$ be $A_{n}$-spaces. According to Stasheff [22, II, Definition 4.1], a map $f: X \rightarrow Y$ is called an $A_{n}$-homomorphism if $f \mu_{i}^{X}=\mu_{i}^{Y}\left(1_{K_{i}} \times f^{i}\right)$ for $1 \leq i \leq n$, where $\left\{\mu_{i}^{X}\right\}_{1 \leq i \leq n}$ and $\left\{\mu_{i}^{Y}\right\}_{1 \leq i \leq n}$ are $A_{n}$-forms on $X$ and $Y$, respectively.

Definition 4.6. Let $n \geq 1$. Assume that $X$ and $Y$ are $B_{n}$-spaces with $B_{n}$-structures $\left\{\varphi_{i}^{X}\right\}_{1 \leq i \leq n}$ and $\left\{\varphi_{i}^{Y}\right\}_{1 \leq i \leq n}$, respectively. An $A_{n}$-homomorphism $f: X \rightarrow Y$ is called a $B_{n^{-}}$-homomorphism if $f \varphi_{i}^{X}=\varphi_{i}^{Y}\left(1_{W_{i}} \times f^{i}\right)$ for $1 \leq i \leq n$.

Example 4.7. Let $(\widetilde{X}, \rho, X)$ be a covering space. If $X$ is a $B_{n}$-space, then $\widetilde{X}$ is also a $B_{n}$-space so that the projection $\rho: \widetilde{X} \rightarrow X$ is a $B_{n}$-homomorphism for $n \geq 1$.

Proof. We give an outline of the proof. Since the result is clear for $n=1$, we assume $n>1$. Let $\left\{\mu_{i}\right\}_{1 \leq i \leq n}$ and $\left\{\varphi_{i}\right\}_{1 \leq i \leq n}$ be an $A_{n}$-form and a $B_{n}$-structure on $X$, respectively.

Put $g_{i}=\mu_{i}\left(1_{K_{i}} \times \rho^{i}\right)$ for $1 \leq i \leq n$. Let $\alpha \in \pi_{1}\left(K_{i} \times \widetilde{X}^{i}\right)$. Since $\pi_{1}\left(K_{i} \times \widetilde{X}^{i}\right)$ $\cong \pi_{1}(\widetilde{X})^{i}$, we can write $\alpha=\left(a_{1}, \ldots, a_{i}\right)$ with $a_{j} \in \pi_{1}(\widetilde{X})$ for $1 \leq j \leq i$. Let $\mu_{i}^{\prime}: K_{i} \times X^{i} \rightarrow X$ be defined by $\mu_{i}^{\prime}\left(b, x_{1}, \ldots, x_{i}\right)=\left(\cdots\left(\left(x_{1} x_{2}\right) x_{3}\right) \cdots\right) x_{i}$. Since $X$ is an $H$-space and $\mu_{i} \simeq \mu_{i}^{\prime}$, we have $g_{i \#}(\alpha)=\rho_{\#}\left(a_{1}\right)+\cdots+\rho_{\#}\left(a_{i}\right)=$ $\rho_{\#}\left(a_{1} * \cdots * a_{i}\right) \in \rho_{\#}\left(\pi_{1}(\widetilde{X})\right)$, where + and $*$ denote the multiplications of $\pi_{1}(X)$ and $\pi_{1}(\tilde{X})$, respectively. Then $g_{i \#}\left(\pi_{1}\left(K_{i} \times \widetilde{X}^{i}\right)\right) \subset \rho_{\#}\left(\pi_{1}(\tilde{X})\right)$, and so we have a lifting $\widetilde{\mu}_{i}: K_{i} \times \widetilde{X}^{i} \rightarrow \widetilde{X}$ with $\rho \widetilde{\mu}_{i}=g_{i}$ for $1 \leq i \leq n$ (cf. [12, Chapter III, Section 16, Theorem 16.2]).

In a similar way, we have a map $\widetilde{\varphi}_{i}: W_{i} \times \widetilde{X}^{i} \rightarrow \widetilde{X}$ with $\rho \widetilde{\varphi}_{i}=\varphi_{i}\left(1_{W_{i}} \times \rho^{i}\right)$ for $1 \leq i \leq n$. From the uniqueness of lifting, $\left\{\widetilde{\mu}_{i}\right\}_{1 \leq i \leq n}$ and $\left\{\widetilde{\varphi}_{i}\right\}_{1 \leq i \leq n}$ are an $A_{n}$-form and a $B_{n}$-structure on $\widetilde{X}$, respectively. 
Consider the double suspension $\Sigma^{2}:\left(S^{2 m-1}\right)_{p}^{\wedge} \rightarrow \widetilde{\Omega}^{2}\left(S^{2 m+1}\right)_{p}^{\wedge}$ which is the double adjoint of the identity $1_{\left(S^{2 m+1}\right)_{p}^{\wedge}}$ on $\left(S^{2 m+1}\right)_{p}^{\wedge} \simeq \Sigma^{2}\left(S^{2 m-1}\right)_{p}^{\wedge}$ for $m \geq 1$, where $p$ is a prime and $Y_{p}^{\wedge}$ denotes the $p$-completion of the space $Y$ in the sense of Bousfield-Kan [5, Chapter VI, Section 6]. By Proposition 4.3 and Remark 4.5, we deduce that $\widetilde{\Omega}^{2}\left(S^{2 m+1}\right)_{p}^{\wedge}$ is a $B_{\infty}$-space.

According to Stasheff [22, I, Theorem 17], $\left(S^{2 m-1}\right)_{p}^{\wedge}$ is an $A_{p-1}$-space such that $\Sigma^{2}:\left(S^{2 m-1}\right)_{p}^{\wedge} \rightarrow \widetilde{\Omega}^{2}\left(S^{2 m+1}\right)_{p}^{\wedge}$ is an $A_{p-1}$-homomorphism.

Example 4.8. Let $p$ be a prime. Then $\left(S^{2 m-1}\right)_{p}^{\wedge}$ is a $B_{p-1}$-space such that the double suspension $\Sigma^{2}:\left(S^{2 m-1}\right)_{p}^{\wedge} \rightarrow \widetilde{\Omega}^{2}\left(S^{2 m+1}\right)_{p}^{\wedge}$ is a $B_{p-1}$-homomorphism for $m \geq 1$.

Proof. Since the result is clear for $p=2$, we consider the case of $p>2$. As in the proof of [22, I, Theorem 17], we assume that $\left(S^{2 m-1}\right)_{p}^{\wedge}$ is a subspace of $\widetilde{\Omega}^{2}\left(S^{2 m+1}\right)_{p}^{\wedge}$ and $\Sigma^{2}:\left(S^{2 m-1}\right)_{p}^{\wedge} \rightarrow \widetilde{\Omega}^{2}\left(S^{2 m+1}\right)_{p}^{\wedge}$ is the inclusion.

For simplicity, we write $X=\left(S^{2 m-1}\right)_{p}^{\wedge}$ and $Y=\widetilde{\Omega}^{2}\left(S^{2 m+1}\right)_{p}^{\wedge}$. Let $\left\{\kappa_{i}\right\}_{i \geq 1}$ be a $B_{\infty}$-structure on $Y$. By induction on $i$, we construct a $B_{p-1}$-structure $\left\{\varphi_{i}\right\}_{1 \leq i \leq p-1}$ on $X$ with $\Sigma^{2} \varphi_{i}=\kappa_{i}\left(1_{W_{i}} \times\left(\Sigma^{2}\right)^{i}\right)$ for $1 \leq i \leq p-1$.

Put $\varphi_{1}(*, x)=x$ for $x \in X$. Assume inductively that $\left\{\varphi_{j}\right\}_{1 \leq j<i}$ is constructed. Let $F_{i}=\partial W_{i} \times X^{i} \cup W_{i} \times X^{[i]}$, where $Z^{[i]}$ denotes the $i$-fold fat wedge of a space $Z$ given by

$$
Z^{[i]}=\left\{\left(z_{1}, \ldots, z_{i}\right) \in Z^{i} \mid z_{j}=* \text { for some } j \text { with } 1 \leq j \leq i\right\} \quad \text { for } i \geq 1 .
$$

Then we have $\left(W_{i} \times X^{i}\right) / F_{i} \simeq\left(S^{2 m i-1}\right)_{p}^{\wedge}$.

Define $\widetilde{\varphi}_{i}: W_{i} \times X^{i} \rightarrow Y$ by $\widetilde{\varphi}_{i}=\kappa_{i}\left(1_{W_{i}} \times\left(\Sigma^{2}\right)^{i}\right)$. By inductive hypothesis, we have $\widetilde{\varphi}_{i}\left(F_{i}\right) \subset X$. Then the obstructions to obtain $\varphi_{i}: W_{i} \times X^{i} \rightarrow X$ with $\Sigma^{2} \varphi_{i} \simeq \widetilde{\varphi}_{i}$ rel $F_{i}$ appear in the following cohomology groups:

$$
H^{k}\left(W_{i} \times X^{i}, F_{i} ; \pi_{k}(Y, X)\right) \cong \widetilde{H}^{k}\left(\left(S^{2 m i-1}\right)_{p}^{\wedge} ; \pi_{k}(Y, X)\right) \quad \text { for } k \geq 1
$$

(cf. [12, p. 197, E.6]). Now, (4.13) is non-trivial only if $k=2 m i-1 \leq 2 m p-2 m-1 \leq$ $2 m p-3$. On the other hand, $\pi_{k}(Y, X)=0$ for $k \leq 2 m p-3$ by Toda [27, Proposition 13.1]. This implies that (4.13) is trivial for any $k$, and we have a map $\varphi_{i}$. From the homotopy extension property, we have a map $\widetilde{\kappa}_{i}: W_{i} \times Y^{i} \rightarrow Y$ with $\widetilde{\kappa}_{i} \simeq \kappa_{i}$ rel $\partial W_{i} \times Y^{i} \cup W_{i} \times Y^{[i]}$ and $\Sigma^{2} \varphi_{i}=\widetilde{\kappa}_{i}\left(1_{W_{i}} \times\left(\Sigma^{2}\right)^{i}\right)$. This completes the induction, and we have a $B_{p-1}$-structure $\left\{\varphi_{i}\right\}_{1 \leq i \leq p-1}$ on $X$.

Hemmi-Kawamoto [9, Definition 3.1] introduced another type of higher homotopy commutativity of $A_{n}$-spaces using the permuto-associahedra $\left\{K P_{i}\right\}_{1 \leq i \leq n}$. Let $X$ be an $A_{n}$-space with an $A_{n}$-form $\left\{\mu_{i}\right\}_{1 \leq i \leq n}$ for $n \geq 1$. Then $X$ is called 
an $A C_{n}$-space if there is a family $\left\{\nu_{i}: K P_{i} \times X^{i} \rightarrow X\right\}_{1 \leq i \leq n}$ of maps with the following relations:

$$
\begin{aligned}
& \nu_{1}(*, x)=x \\
& \nu_{i}\left(\varepsilon^{\left(\alpha_{1}, \ldots, \alpha_{m}\right)}\left(a, b_{1}, \ldots, b_{m}\right), x_{1}, \ldots, x_{i}\right) \\
& =\mu_{m}\left(a, \nu_{t_{1}}\left(b_{1}, x_{\alpha_{1}(1)}, \ldots, x_{\alpha_{1}\left(t_{1}\right)}\right), \ldots, \nu_{t_{m}}\left(b_{m}, x_{\alpha_{m}(1)}, \ldots, x_{\alpha_{m}\left(t_{m}\right)}\right)\right) \\
& \quad \text { for }\left(\alpha_{1}, \ldots, \alpha_{m}\right) \in \mathbb{A}_{i}^{\left(t_{1}, \ldots, t_{m}\right)} \text { with } m \geq 2 ; \\
& \nu_{i}\left(a, x_{1}, \ldots, x_{j-1}, *, x_{j+1}, \ldots, x_{i}\right) \\
& \quad=\nu_{i-1}\left(\omega_{j}(a), x_{1}, \ldots, x_{j-1}, x_{j+1}, \ldots, x_{i}\right) \quad \text { for } 1 \leq j \leq i .
\end{aligned}
$$

Remark 4.9. (1) An $A C_{1}$-space is just a space. Since $\nu_{2}\left(\varepsilon^{((1),(2))}(*, *, *), x_{1}, x_{2}\right)$ $=x_{1} x_{2}$ and $\nu_{2}\left(\varepsilon^{((2),(1))}(*, *, *), x_{1}, x_{2}\right)=x_{2} x_{1}$ for $x_{1}, x_{2} \in X$, an $A C_{2}$-space is the same as a homotopy commutative $H$-space.

(2) When $X$ is a topological monoid, $X$ is an $A C_{n}$-space if and only if it is a $C_{n}$-space in the sense of Williams [28, Definition 5].

Proof of Theorem B. We work by induction on $n$. The result is clear for $n=1$. Assume inductively that the result is proved for any $n^{\prime}<n$.

Let $X$ be a $B_{n}$-space with a $B_{n}$-structure $\left\{\varphi_{i}\right\}_{1 \leq i \leq n}$. By inductive hypothesis, $X$ is an $A C_{n-1}$-space with an $A C_{n-1}$-structure $\left\{\nu_{i}\right\}_{1 \leq i \leq n-1}$. From Theorem $\mathrm{A}$ and Remark 3.2, we can define $\nu_{n}: K P_{n} \times X^{n} \rightarrow X$ by

$$
\begin{aligned}
& \nu_{n}\left(\iota^{\left(\alpha_{1}, \ldots, \alpha_{m}\right)}\left(a, b_{1}, \ldots, b_{m}\right), x_{1}, \ldots, x_{n}\right) \\
& =\varphi_{m}\left(a, \nu_{t_{1}}\left(b_{1}, x_{\alpha_{1}(1)}, \ldots, x_{\alpha_{1}\left(t_{1}\right)}\right), \ldots, \nu_{t_{m}}\left(b_{m}, x_{\alpha_{m}(1)}, \ldots, x_{\alpha_{m}\left(t_{m}\right)}\right), x_{n}\right) \\
& \text { for }\left(\alpha_{1}, \ldots, \alpha_{m}\right) \in \mathbb{A}_{n-1}^{\left(t_{1}, \ldots, t_{m}\right)} \text { with } m \geq 1
\end{aligned}
$$

Figure 12. The $B_{3}$-structure on $X$.

(see Figure 12). From the proof of Theorem A, we see that $\left\{\nu_{i}\right\}_{1 \leq i \leq n}$ is an $A C_{n^{-}}$ structure on $X$. 
Let $p$ be a prime. An $H$-space $X$ is called $p$-Postnikov if there is an integer $l_{X} \geq 1$ such that $\pi_{j}(X)$ is finitely generated over the $p$-adic integers $\mathbb{Z}_{p}^{\wedge}$ for $1 \leq$ $j \leq l_{X}$ and $\pi_{j}(X)=0$ for $j>l_{X}$. For example, Eilenberg-Mac Lane spaces $K\left(\mathbb{Z}_{p}^{\wedge}, m\right)$ and $K\left(\mathbb{Z} / p^{i}, m\right)$ are $p$-Postnikov $H$-spaces for $i, m \geq 1$.

Remark 4.10. By the result of McGibbon-Neisendorfer [19, Theorem 1], if $X$ is a connected $p$-Postnikov $H$-space whose cohomology $H^{*}\left(X ; \mathbb{F}_{p}\right)$ is finite-dimensional, then $X$ is homotopy equivalent to a $p$-completed torus.

Let $\left(\mathbb{C} P^{\infty}\right)_{p}^{\wedge}$ denote the $p$-completion of the infinite-dimensional complex projective space. Its cohomology is given by $H^{*}\left(\left(\mathbb{C} P^{\infty}\right)_{p}^{\wedge} ; \mathbb{F}_{p}\right) \cong \mathbb{F}_{p}[u]$ with $\operatorname{deg} u=2$. Denote the homotopy fiber of the map $f_{t}:\left(\mathbb{C} P^{\infty}\right)_{p}^{\wedge} \rightarrow K(\mathbb{Z} / p, 2 t)$ corresponding to the class $u^{t} \in H^{2 t}\left(\left(\mathbb{C} P^{\infty}\right)_{p}^{\wedge} ; \mathbb{F}_{p}\right)$ by $Y_{t}$ for $t \geq 1$. Put $X_{t}=\widetilde{\Omega} Y_{t}$.

Remark 4.11. (1) $X_{t}$ is a $p$-Postnikov $H$-space.

(2) $Y_{t}$ is an $H$-space if and only if $t=p^{i}$ for some $i \geq 1$.

By Remarks 4.5 and 4.9 , we have the following example:

Example 4.12 ([11, Propositions 5.3 and 5.5]). (1) If $t=1$ or $t \equiv 0 \bmod p$, then $X_{t}$ is a $B_{\infty}$-space.

(2) If $1<t<p$, then $X_{t}$ is a $B_{t-1}$-space, but not an $A C_{t}$-space.

(3) If $t>p$ with $t \not \equiv 0 \bmod p$, then $X_{t}$ is an $A C_{\infty}$-space, which is also a $B_{t-1}$-space, but not a $B_{t}$-space.

From Theorem B, all results stated for $A C_{n}$-spaces also hold for $B_{n}$-spaces (cf. [9], [10] and [15]).

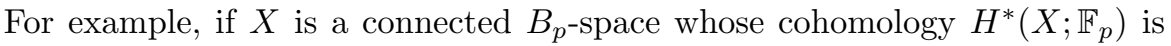
finitely generated as an algebra over the Steenrod algebra $\mathscr{A}_{p}^{*}$, then $X_{p}^{\wedge}$ is a $p$ Postnikov $H$-space by [15, Theorem B]. Moreover, if $H^{*}\left(X ; \mathbb{F}_{p}\right)$ is finitely generated as an algebra over $\mathbb{F}_{p}$, then $X_{p}^{\wedge}$ is homotopy equivalent to a finite product of $\left(S^{1}\right)_{p}^{\wedge} \mathrm{s},\left(\mathbb{C} P^{\infty}\right)_{p}^{\wedge} \mathrm{s}$ and $B \mathbb{Z} / p^{i}$ s with $i \geq 1$ using [9, Theorem B]. On the other hand, $\left(S^{2 m-1}\right)_{p}^{\wedge}$ is a $B_{p-1}$-space which is not $p$-Postnikov for any $m>1$ by Example 4.8 and Remark 4.10.

\section{Acknowledgements}

This research was partially supported by Grant-in-Aid for Scientific Research (No. 24740053), Japan Society for the Promotion of Science. The content of the paper was first presented to a conference on algebraic topology at Shinshu University in March 2011. The author is grateful to the organizers for their kind invitation and hospitality. We would also like to thank the referee for many useful comments. 


\section{References}

[1] J. Aguadé, Decomposable free loop spaces, Canad. J. Math. 39 (1987), 938-955. Zbl 0644.55008 MR 0915024

[2] D. Bar-Natan, Non-associative tangles, in Geometric topology (Athens, GA, 1993), AMS/IP Stud. Adv. Math. 2, Part 1, Amer. Math. Soc., Providence, RI, 1997, 139-183. Zbl 0888.57008 MR 1470726

[3] D. Bar-Natan and A. Stoimenow, The fundamental theorem of Vassiliev invariants, in Geometry and physics (Aarhus, 1995), Lecture Notes in Pure Appl. Math. 184, Dekker, New York, 1997, 101-134. Zbl 0878.57004 MR 1423158

[4] R. Bott and C. Taubes, On the self-linking of knots, J. Math. Phys. 35 (1994), 5247-5287. Zbl 0863.57004 MR 1295465

[5] A. K. Bousfield and D. M. Kan, Homotopy limits, completions and localizations, Lecture Notes in Math. 304, Springer, Berlin, 1972. Zbl 0259.55004 MR 0365573

[6] S. L. Devadoss, A space of cyclohedra, Discrete Comput. Geom. 29 (2003), 61-75. Zbl 1027.52007 MR 1946794

[7] I. M. Gel'fand, M. M. Kapranov and A. V. Zelevinsky, Newton polytopes of the classical resultant and discriminant, Adv. Math. 84 (1990), 237-254. Zbl 0721.33002 MR 1080979

[8] Y. Hemmi, Higher homotopy commutativity of $H$-spaces and the mod $p$ torus theorem, Pacific J. Math. 149 (1991), 95-111. Zbl 0691.55007 MR 1099785

[9] Y. Hemmi and Y. Kawamoto, Higher homotopy commutativity of $H$-spaces and the permuto-associahedra, Trans. Amer. Math. Soc. 356 (2004), 3823-3839. Zbl 1064.55005 MR 2058507

[10] Higher homotopy commutativity and cohomology of finite $H$-spaces, in Proceedings of the Nishida Fest (Kinosaki, 2003), Geom. Topol. Monogr. 10, Geom. Topol. Publ., Coventry, 2007, 167-186. Zbl 1117.55004 MR 2402783

[11] Higher homotopy commutativity and the resultohedra, J. Math. Soc. Japan 63 (2011), 443-471. Zbl 1222.55009 MR 2793107

[12] S. T. Hu, Homotopy theory, Pure Appl. Math. 8, Academic Press, New York, 1959. Zbl 0088.38803 MR 0106454

[13] M. M. Kapranov, The permutoassociahedron, Mac Lane's coherence theorem and asymptotic zones for the KZ equation, J. Pure Appl. Algebra 85 (1993), 119-142. Zbl 0812.18003 MR 1207505

[14] M. M. Kapranov and V. A. Voevodsky, 2-categories and Zamolodchikov tetrahedra equations, in Algebraic groups and their generalizations: quantum and infinite-dimensional methods (University Park, PA, 1991), Proc. Sympos. Pure Math. 56, Part 2, Amer. Math. Soc., Providence, RI, 1994, 177-259. Zbl 0809.18006 MR 1278735

[15] Y. Kawamoto, Higher homotopy commutativity of $H$-spaces and homotopy localizations, Pacific J. Math. 231 (2007), 103-126. Zbl 1155.55002 MR 2304624

[16] S. Mac Lane, Natural associativity and commutativity, Rice Univ. Studies 49 (1963), 28-46. Zbl 0244.18008 MR 0170925

[17] M. Markl, Simplex, associahedron, and cyclohedron, in Higher homotopy structures in topology and mathematical physics (Poughkeepsie, NY, 1996), Contemp. Math. 227, Amer. Math. Soc., Providence, RI, 1999, 235-265. Zbl 0919.18003 MR 1665469

[18] J. P. May, The geometry of iterated loop spaces, Lecture Notes in Math. 271, Springer, Berlin, 1972. Zbl 0244.55009 MR 0420610

[19] C. A. McGibbon and J. A. Neisendorfer, On the homotopy groups of a finite-dimensional space, Comment. Math. Helv. 59 (1984), 253-257. Zbl 0538.55010 MR 0749108 
[20] R. J. Milgram, Iterated loop spaces, Ann. of Math. (2) 84 (1966), 386-403. Zbl 0145.19901 MR 0206951

[21] V. Reiner and G. M. Ziegler, Coxeter-associahedra, Mathematika 41 (1994), 364-393. Zbl 0822.52007 MR 1316615

[22] J. D. Stasheff, Homotopy associativity of $H$-spaces I, II, Trans. Amer. Math. Soc. 108 (1963), 275-292; ibid. 108 (1963), 293-312. Zbl 0114.39402 MR 0158400

[23] H H-spaces from a homotopy point of view, Lecture Notes in Math. 161, Springer, Berlin, 1970. Zbl 0205.27701 MR 0270372

[24] From operads to "physically" inspired theories, in Operads: Proceedings of Renaissance Conferences (Hartford, CT/Luminy, 1995), Contemp. Math. 202, Amer. Math. Soc., Providence, RI, 1997, 53-81. Zbl 0872.55010 MR 1436913

[25] M. Sugawara, A condition that a space is group-like, Math. J. Okayama Univ. 7 (1957), 123-149. Zbl 0091.37201 MR 0097066

[26] - On the homotopy-commutativity of groups and loop spaces, Mem. Coll. Sci. Univ. Kyoto Ser. A Math. 33 (1960/1961), 257-269. Zbl 0113.16903 MR 0120645

[27] H. Toda, Composition methods in homotopy groups of spheres, Ann. of Math. Stud. 49, Princeton Univ. Press, Princeton, NJ, 1962. Zbl 0101.40703 MR 0143217

[28] F. D. Williams, Higher homotopy-commutativity, Trans. Amer. Math. Soc. 139 (1969), 191-206. Zbl 0185.27103 MR 0240818

[29] G. M. Ziegler, Lectures on polytopes, Grad. Texts in Math. 152, Springer, New York, 1995. Zbl 0823.52002 MR 1311028 